\title{
An annotated catalogue of the type material of Elateroidea Leach, 1815 (Coleoptera) deposited in the Coleoptera collection of the Museum of Zoology of the University of São Paulo, Brazil
}

\section{Authors: Vinicius S. Ferreira}

This is a postprint of an article that originally appeared in Zootaxa on March 25, 2015 and the final version can be found here https://doi.org/10.11646/zootaxa.3937.2.3.

Ferreira, Vinicius S. (2015). An annotated catalogue of the type material of Elateroidea Leach, 1815 (Coleoptera) deposited in the Coleoptera collection of the Museum of Zoology of the University of São Paulo, Brazil. Zootaxa, 3937(2), 263-310. doi:10.11646/zootaxa.3937.2.3

Made available through Montana State University's $\underline{\text { ScholarWorks }}$ scholarworks.montana.edu 


\title{
An annotated catalogue of the type material of Elateroidea Leach, 1815 (Coleoptera) deposited in the Coleoptera collection of the Museum of Zoology of the University of São Paulo, Brazil
}

\author{
VINICIUS DE SOUZA FERREIRA \\ Museu de Zoologia da Universidade de São Paulo (MZSP), Setor de Entomologia, Laboratório de Sistemática, Evolução e Bionomia
} de Coleoptera, Avenida Nazaré, 481, Ipiranga, CEP: 04263-000, São Paulo-SP, Brazil.E-mail: vinicius.sfb@gmail.com

\begin{abstract}
The Museum of Zoology of the University of São Paulo (MZSP) houses one of the most important Coleoptera collections of Brazil and Neotropical Region with nearly 900,000 adult mounted material and about 1,500,000 specimens to be mounted. The superfamily Elateroidea Leach, 1815 (including Cantharoidea) comprises about 24,077 described species in 17 families. The MZSP owns type material of Brachypsectridae LeConte \& Horn, 1883, Cantharidae, 1856 (1815), Cerophytidae Latreille, 1834, Elateridae Leach 1815, Eucnemidae Eschscholtz, 1829, Lampyridae Rafinesque, 1815, Lycidae Laporte, 1836, Phengodidae LeConte, 1861 and Rhinorhipidae Lawrence, 1988. This catalogue includes type material of 166 species distributed in 69 genera. Among 1,223 type specimens, are 86 holotypes, 1,133 paratypes, 2 allotypes, 1 lectotype and 1 paralectotype.
\end{abstract}

Key words: Elateridae, Lycidae, Phengodidae, Cantharidae, Eucnemidae

\section{Resumo}

A coleção de Coleoptera do Museu de Zoologia da Universidade de São Paulo (MZSP) abriga uma das mais importantes coleções do Brasil e da região Neotropical com aproximadamente 900,000 adultos montados e cerca de 1.500 .000 espécimens a serem montados. A superfamília Elateroidea Leach, 1815 (incluindo Cantharoidea) compreende cerca de 24.077 espécies descritas em 17 famílias. O MZSP possui material-tipo de Brachypsectridae LeConte \& Horn, 1883, Cantharidae, 1856 (1815), Cerophytidae Latreille, 1834, Elateridae Leach 1815, Eucnemidae Eschscholtz, 1829, Lampyridae Rafinesque, 1815, Lycidae Laporte, 1836, Phengodidae LeConte, 1861 e Rhinorhipidae Lawrence, 1988. Este catálogo inclui material-tipo de 166 espécies distribuídas em 69 gêneros. Dentre 1223 tipos, há 86 holótipos, 1133 parátipos, 2 alótipos, 1 lectótipo e 1 paralectótipo.

Palavras chave: Elateridae, Lycidae, Phengodidae, Cantharidae, Eucnemidae

\section{Introduction}

The Museum of Zoology of the University of São Paulo (MZSP) is considered one of the most traditional institutions in Brazil and its collections have been very useful to a more complete and broad understanding of the animal evolution and diversity studies in Brazil and in Neotropical region (Taddei et al., 1999; Marinoni \& Marinoni, 2012).

With one of the most important collections of Brazil and Neotropical Region the Coleoptera Collection of the Museum of Zoology houses nearly 900,000 adults of beetles mounted and about 1,500,000 specimens to be mounted (Costa 1999; Costa et al. 2000) and most of the unidentified material in the former collection of Richard Von Diringshofen (Costa et al. 2000; Ferreira et al. in prep). The MZSP also owns a very important immature collection, the Immature Coleoptera Collection (CIC-MZSP), with about 51,000 species maintained on $70 \%$ ethyl alcohol (Costa 2010; Barbosa et al. 2013). Up to now no list of type material of Elateroidea deposited in the MZSP has been published. 
The superfamily Elateroidea Leach, 1815 (including Cantharoidea) comprises about 24,077 described species in 17 families (Bouchard et al. 2011; Slipinski et al. 2011). Cantharoidea was included in Elateroidea by Lawrence (1988) and thereafter this classification has been accepted and corroborated by many other authors (e.g. Branham \& Wenzel 2000; Hunt et al. 2007; Bocakova et al. 2007; Lawrence et al. 2010; Kundrata \& Bocak 2011; Lawrence et al. 2011; Kundrata et al. 2014).

The currently accepted Elateroidea families (sensu Bouchard et al. 2011) in MZSP collections are: Artematopodidae Lacordaire, 1857, Brachypsectridae LeConte \& Horn, 1883, Cantharidae, 1856 (1815) Cerophytidae Latreille, 1834, Drilidae Blanchard 1845, Elateridae Leach 1815, Eucnemidae Eschscholtz, 1829, Lampyridae Rafinesque, 1815, Lycidae Laporte, 1836, Omalisidae Lacordaire, 1857, Omethidae LeConte, 1861, Phengodidae LeConte, 1861, Plastoceridae Crowson, 1972, Rhagophthalmidae Olivier, 1907, Rhinorhipidae Lawrence, 1988, Teleugesidae Leng, 1920 and Throscidae Laporte, 1840. The MZSP owns type material of Brachypsectridae, Cerophytidae, Cantharidae, Elateridae, Eucnemidae, Lampyridae, Lycidae, Phengodidae and Rhinorhipidae. Most (55 species) of the Elateroidea type specimens deposited in MZSP were described by Cleide Costa. This catalogue aims to list the type material of Elateroidea beetles deposited in the MZSP and to provide label information and preservation status of each specimen.

\section{Material and methods}

All drawers of the families previously cited were examined and information about labels and the condition of type material are portrayed. The conservation status and other pertinent comments are given after the label description. Specimens considered in good condition are those in suitable status for taxonomic and morphological study with none or few parts of the body broken.

Information in this catalogue is given in the following order: family, genus and author; subgenus and author; species name (as well as subspecies), authorship, publication year, page numbers, type category, number of specimens followed by sex ( $\delta$ male, $q$ female, unknown sex). Label information of each specimen is given as written on the label itself, with each label separated by a semicolon. Additional data on labels are presented between brackets. Detailed data for conservation status is given with comments after labels information. Additional notes are given when necessary after conservation status. The nomenclature of families follows Bouchard et al. (2011). All taxa are presented alphabetically by family, then also by genus and species.

\section{Results}

This catalogue includes type material of 166 species distributed in 69 genera. Among 1,223 type specimens, are 86 holotypes, 1,133 paratypes, 2 allotypes, 1 lectotype and 1 paralectotype.

\section{Brachypsectridae LeConte \& Horn, 1883}

\section{Genus Brachypsectra LeConte, 1874}

Brachypsectra vivafosile Woodruff, 2002: 161

Paratypes: 1 ठ . DOMINICAN REPUBLIC: Prov. of Pedernales, Cabo, Rojo, Alcoa, 20-24-VI-1999, blacklight trap, R.M. Baranowski, R.E. Woodruff; [on yellow] Paratype, Brachypsectra vivafosile, R. E. Woodruff 2004.

Conservation status: Good condition, genitalia in microvial with glycerin under specimen.

\section{Cantharidae Imhoff, 1856 (1815)}

Genus Discodon Gorham, 1881

Discodon dureti Wittmer, 1945: 314 
Paratype: 1 ठ․ Cotype [on red]; Cotype [on red]; El Naranjo, R. de La F. Salta, Argentina, I-44, Duret-Martínez; Discodon dureti Wittmer det W. Wittmer.

Conservation status: Good condition; specimen glued on card.

Discodon saltense Wittmer 1945: 316

Paratype: 1 ठै. [on red] Cotype; [on red] Cotype; El Naranjo, R. de La F. Salta, Argentina, I-44, Duret-Martínez; Discodon saltensis Wittmer det W. Wittmer.

Conservation status: Good condition; specimen glued on card.

Discodon martinezi Wittmer, 1945: 317

Paratype: 1 o. [on red] Cotype; [on red] Cotype; El Naranjo, R. de La F. Salta, Argentina, I-44, Duret—Martínez; Discodon martinezi Wittmer det W. Wittmer.

Conservation status: Good condition; specimen glued on card.

\section{Genus Oontelus Solier, 1849}

Oontelus reichardti Wittmer, 1967: 427

Holotype: $1 \delta$. [on red] Holotypus; São Paulo, (Ipiranga) SP, 5.VIII.1907, H. Lüderwaldt col.; 9205; Oontelus reichardti Wittmer det W. Wittmer.

Conservation status: Good condition; specimen glued on triangle with its abdomen.

Oontelus nodulicornis Wittmer, 1966: 416

Paratypes: $2 \delta^{\lambda}$. Paratypus; Brasilien, Nova Teutônia, $27^{\circ} 11^{\prime} 852^{\circ} 23$ [the last number is illegible] Fritz Plaumann, 3.500m, 9/8/1948; Oontelus nodulicornis Wittmer det. W. Wittmer.

Conservation status: Good condition; both specimens glued on cards, one with abdomen and genitalia glued on second card under the specimen.

\section{Genus Paramonius Wittmer, 1963}

Paramonius campbelli Brancucci, 1983: 362

Holotype: 1 ○े. Brazil, DF, Parque Nacional, 1.000m, 9.III.1970, J.M \& B.A. Campbell.

Conservation status: Good condition.

\section{Genus Silis Charpentier, 1825}

\section{Subgenus Peltariosilis Wittmer, 1952}

\section{Peltariosilis amapaensis Wittmer, 1966: 414}

Holotype: $1 \delta^{\Uparrow}$ [on red] Holotypus; Ex.-Coleção M. Alvarenga; Oiapoque, Amapá-Brasil, V.1959, M. Alvarenga col.; Peltariosilis amapaensis Wittmer det. W. Wittmer.

Conservation status: Bad condition; both antennae missing. Specimen glued on triangle by right elytra. Paratypes: 4 ठ․ [on red] Paratypus; Ex.-Coleção M. Alvarenga; Oiapoque, Amapá- Brasil, V.1959, M. Alvarenga col.; Peltariosilis amapaensis Wittmer det. W. Wittmer.

Conservation status: Bad condition; all specimens with both antennae missing. All specimens glued on triangle by right elytra.

Additional notes: the species was described in the subgenus Peltariosilis by Wittmer (1952). Constantin (2010) considered Peltariosilis a distinct genus. 
Genus Phytocerum Costa, Vanin, Lawrence \& Ide, 2003

Phytocerum allenum Costa, Vanin, Lawrence \& Ide, 2003: 390

Paratype: $1 \delta$. Nicaragua: $50 \mathrm{~km}$. E, of Matagalpa, El Coyobar, 15 May 1991; S. Hue, en trampa luminies 6V, para Lutzomyia; Phytocerum alleni Costa et al. 2003, Paratype F, C. Costa det. 2003; [on red] Parátipo; Retido SDSU.

Conservation status: Good condition, abdomen glued on a card under specimen. Genitalia in microvial with glycerin.

Phytocerum belloi Costa, Vanin, Lawrence \& Ide, 2003: 391

Holotype: 1 ô. Águas Vermelhas, MG-Brasil, XII -1997, Col. A. Bello; Phytocerum belloi Costa et al. 2003, Holotype M. C. Costa det. 2003; [on red] Holotype.

Conservation status: Good condition, genitalia in microvial with glycerin under specimen.

Paratype: 1 ㅇ. Brasil: BA: MARACAS, Faz. M. Inácia-Mata Cipó, noite -luz XI 1990, S.T.P. AMARANTE COL; Phytocerum belloi Costa et al, 2003, Paratype F, C. Costa det. 2003; [on red] Paratipo.

Conservation status: Good condition.

Paratype: 1 ‥ Idem, 25.XI.1990.

Conservation status: Idem, right antenna glued on a card under specimen.

Phytocerum birai Costa, Vanin, Lawrence \& Ide, 2003: 392

Paratype: 1 ठ. Brazil, Goias, Jataí, XI.1972, F. M. Oliveira; Phytocerum birai Costa et al. 2003, Paratype M, C. Costa det. 2003; [on red] Paratype; Retido CNCI.

Conservation status: Good condition, specimen glued on a card, genitalia in microvial with glycerin under specimen.

Phytocerum boliviense (Golbach, 1983c): 393

Paratype: 1 ठ․ Bolivia, Dpto Santa Cruz, Est, Exp. Gral. Saavedra, 6-12.VII.1972, C. Porter L. Stange; ठ; [on yellow] Parátipo; Phytocerum boliviense (Golbach, 1983), C. Costa det. 2003; Cerophytum boliviensis n. sp. R., Golbach det.; Doação Golbach; [on red] Paratype.

Conservation status: Good condition, abdomen glued on a card under specimen, genitalia in microvial with glycerin under specimen.

Phytocerum distinguendum (Soares \& Peracchi, 1964): 130

Holotype: 1 [unknown sex]. Est. Minas Gerais, Água Suja, Muschow 1918; M; Types; Phytocerum distinguedum (Soares \& Peracchi, 1964), C. Costa det. 2003; Cerophytum distinguendum B.A.M. Soares \& A. Peracchi det. 1962; [on red] Holótipo.

Conservation status: Good condition, part of right antenna glued on a transparent card under specimen.

Phytocerum golbachi Costa, Vanin, Lawrence \& Ide, 2003: 396

Paratypes: 3 [unknown sex] Argentina: Salta Rosario de Lerma, XII-21/23-1983, Malaise Trap, M. Wasbauer coll.; [on red] Parátipo; Phytocerum golbachi; Retido CDAE.

Conservation status: Two specimens in good condition, one with genitalia in microvial with glycerin under specimen; one in bad condition, specimen and genitalia, each one on a microvial.

Paratype: 1 [unknown sex] Idem, 26/27-1983.

Conservation status: Good condition, right antenna broken. 
Phytocerum ingens Costa, Vanin, Lawrence \& Ide, 2003: 397

Holotype: 1 đ̊. Corrego Itá, E. Santo. Br., X. 1954, W. Zikan; M; Phytocerum ingens Costa et al., 2003, Holotype M, C. Costa det. 2003; Cerophytum cayenensse Bonvoulouir, B. A. M. Soares \& A. Peracchi det. 1962; [on red] Holótipo.

Conservation status: Good condition, genitalia on a microvial with glycerin under specimen, right antenna glued on a triangle under specimen.

Paratypes: $1 \delta^{\lambda}, 1$ ㅇ, Idem

Conservation status: Good condition, genitalia and abdomen in microvial with glycerin under specimen.

Phytocerum zikani (Soares \& Peracchi, 1964): 128

Holotype: 1 ภ. Mar de Hespanha, R, Minas-Brazil, 25-10-1910, J. F. Zikán; M; № 12.056, I. O. C. Coleoptera; Typus; Phtocerum zikani (Soares \& Golbach, 1964), C. Costa det. 2003; Cerophytum zikani B. A. M. Soares \& A. Peracchi det. 1962; [on red] Holótipo.

Conservation status: Good condition, both antennae missing, left mesoleg lost. Genitalia in microvial with glycerin under specimen.

Elateridae Leach, 1815

Genus Agriotes Eschscholtz, 1829

Agriotes sagittus Becker, 1956: 79

Paratype: 1 ․ Humboldt co., cal. V.[1]911; Coll. D. by F.W. Nunenm Acher. Van Dyke Collection; [on yellow] Paratype, Agriotes sagittus Becker , No. 6520.

Conservation status: Good condition; specimen glued on white triangle by mesoventrite.

Agriotes vandykei Becker 1956: 90

Paratype: $1 \hat{\jmath}$. Berkeley, Cal., 4-12-1930; H. E. Collector; [on yellow] Paratype, Agriotes vandykei $\widehat{\jmath}$, No. 6524 Becker.

Conservation status: Good condition, genitalia glued on a triangle under specimen.

Paratype: 1 ㅇ. Hills back of Berkeley Cal., 16-22-1919; H.W. Wenzel Collection; [on yellow] Paratype, Agriotes vandykei , , No. 6524 Becker.

Conservation status: Good condition; left antenna missing. Specimen glued on white triangle by mesoventrite.

\section{Genus Alampoides Schwarz, 1906}

Alampoides badius Costa, 1975a: 63

Paratypes: 1 [unknown sex], 1§ึ. Pérou, Movobamba, M. de Mathan, 1888; Museum Paris, 1952, Coll. R. Oberthur; MNHNP; [on red] Alampoides badius parátipo, C. Costa det. 1975.

Conservation status: Good condition; genitalia into microvial with glycerin under specimen. One specimen with two legs and part of an antenna glued on cards under specimen.

\section{Alampoides fulvus Costa, 1975a:63}

Paratype: $1 \delta$. Pérou, Movobamba, M. de Mathan, 1888; Museum Paris, 1952, Coll. R. Oberthur; MNHNP; [on red] Alampoides fulvus parátipo, C. Costa det. 1975.

Conservation status: Good condition; genitalia glued on transparent card under specimen. 
Paratype: 1 [unknown sex]. Pérou, Taranoto, Mai à Aout 1886, M. de Mathan; Museum Paris, 1952, Coll. R. Oberthur; MNHNP; [on red] Alampoides fulvus parátipo, C. Costa det. 1975.

Conservation status: Good condition.

\section{Genus Alaus Eschscholtz, 1829}

\section{Alaus veracruzanus Casari, 1996: 263}

Holotype: $1 \hat{\delta}$. [on red] Holotypus; Calcolepis lucaoti Cand. M; Alaus veracruzanus sp. n., Holotype, S. A. Casari det. 1996; USNM; USNM 93.

Conservation status: Good condition; genitalia into microvial with glycerin under specimen.

Alaus latipennis Casari, 2003: 322

Paratype: 1 đึ. Dominican Rep: Prov., Barahona, nr Filipinas, Larimae Mine: 26-VI-7-VII-1992: R. E. Woodruff, P. E. Skelley, at light; retained FSCA; [on red] paratype; Alaus latipennis Casari, 2003; [on red] Paratype.

Conservation status: Good condition; genitalia into microvial with glycerin under specimen.

Alaus thoracopunctactus Casari, 2003: 325

Paratype: $1 \delta$. Guatemala: Zacapa, 5km S la Unión, 4600, May 5-11.1987, E. Giesbert col.; Alaus plebeus, Candeze, det E. Giesbert; retained FSCA; [on red] paratype; Alaus thoracopunctactus Casari, 2003.

Conservation status: Good condition; genitalia into microvial with glycerin under specimen.

\section{Genus Ampedus Dejean, 1833}

Ampedus varipilis columbianus Brown, 1933: 136

Paratype: 1 [unknown sex]. Paratype labels: Creston, B. C. 20.VI.1931, G. Stace Smith; [on yellow] Paratype, 3466, Ampedus varipilis columbianus Brown.

Conservation status: Good condition.

\section{Genus Anchastus LeConte, 1853}

Anchastus knulli Becker, 1972:121

Paratypes: 1 [unknown sex]. Madera Cyn., Ariz. Pima, Co. ix.8.1963; V.L.Vesterby Collector; [on yellow] Paratype, Anchastus knulli Becker, CNC No. 12.685.

Conservation status: Good condition. Right antenna missing.

\section{Genus Anoplischius Candéze, 1859}

Anoplischius boraceiensis Casari-Chen \& Costa, 1992: 47

Holotype: 1 §. Est. Biol. Boracéia, Salesópolis, SP, 20-22.IV.1988, Exp. MZSP col.; [on red] Holótipo; Anoplischius boraceiensis Casari-Chen \& Costa, 1991; larval exuvia in alcohol.

Conservation status: Good condition.

Paratypes: 2 ô. Idem.

Conservation status: Idem. 
Aptopus luridus Aranda, 1996: 435

Paratype: 1 [unknown sex]. Argentina, Tucuman, Farallón Blanco, Dpto. Burruyacu, 7-8-II-1961, Col. R. Golbach; [on yellow] Paratipo; Aptopus luridus Aranda; [on red] Aptopus luridus Aranda.

Conservation status: Good condition; right antenna broken.

\section{Aptopus maculatus Aranda, 1996:437}

Paratype: 1 [unknown sex]. Chilecito, 15-30/1/69, La Rioja; [on yellow] Paratipo; Aptopus maculatus Aranda; [on red] Aptopus maculatus Aranda.

Conservation status: Good condition; left antenna missing.

Aptopus golbachi Aranda, 1996: 438

Paratype: 1 [unknown sex]. Aptopus golbachi Aranda; Aptopus golbachi det. Aranda'90; Salta Yacocmuya, Capayate, 1950 mts; [on yellow] Paratipo; [on red] Aptopus maculatus Aranda, Paratype.

Conservation status: Good condition; both antennae missing.

\section{Genus Arcanelater Costa, 1975}

Arcanelater spurius (Germar, 1841): 56

Lectotype: 1 [unknown sex]. [on red] Lectotipo; [on green] Porto Alegre, fe220; 17169; spurius Germ.; Arcanelater spurius (Germar, 1841) C. Costa det. 1975; BM.

Conservation status: Good condition.

Paralectotype: 1 [unknown sex]. [on red] Paralectotipo; 41; [on green] Porto Alegre, Jan 220; Nr. 17169; Arcanelater spurius (Germar, 1841) C. Costa det. 1975; BM.

Conservation status: Good condition.

Additional notes: Originaly described in Pyrophorus Billberg, 1820.

Genus Atractosomus Lacordaire, 1857

Atractosomus amazonicus Casari, 2012: 419

Holotype: 1 ठ․ Belém, Pará, Brasil, IX.1964, E. Dente; Atractosomus amazonicus Casari, 2012; [on red] Holótipo. Conservation status: Good condition.

Paratype: 1 ô. Coleção J. Lane; Oter-Amapá, Serranavio, H Bicelli col.; Atractosomus amazonicus Casari, 2012; [on red] Parátipo.

Conservation status: Idem.

Paratype: 1 đ̊. Sta. Isabel do Pará, PA, Brasil, 30.II.1962, J. Bechyné col.; Convênio DZSP-Goeldi; Atractosomus amazonicus Casari, 2012; [on red] Parátipo.

Conservation status: Idem; genitalia into a microvial with glycerin.

\section{Genus Athous Eschscholtz, 1829}

\section{Athous fueguensis Golbach \& Aranda, 1991: 85}

Paratype: 1 [unknown sex]. Athous fueguensis n. sp., Golbach, Aranda; Ensenada, Parque Nac. Ushuaia, Tierra del Fuego-ARG, 83-XI-5, LEG. APS; [on yellow] Parátipo.

Conservation status: Good condition; mounted on a card. 
Paratype: 1 [unknown sex]. Athous fueguensis n. sp., Golbach, Aranda; Pq. Garibaldi, R. Nac. 3, Tierra del Fuego-ARG, 83-X-23. LEG. APS; [on yellow] Parátipo.

Conservation status: Good condition.

\author{
Athous orvus Becker, 1974: 742 \\ Paratype: 1 [unknown sex]. Ste-Foy 23.vii.62, Québec Co.; J.-C. Aubé; [on yellow] Paratype, Athous orvus \\ Becker, CNC No. 5660 . \\ Conservation status: Good condition. \\ Paratype: 1 [unknown sex]. Ste-Foy 19.vii.62, Québec Co.; G. Chantal; [on yellow] Paratype, Athous orvus \\ Becker, CNC No. 5660 . \\ Conservation status: Good condition.
}

\title{
Genus Buckelater Costa, 1973
}

Buckelater argutus Costa, 1973: 35

Holotype: 1 đ..[on red] Holotipo; S. Caraça (Engenho), 800m-Minas-XI [1]961, Kloss, Lenko, Martins \& Silva col.; [on red] Buckelater argutus C. Costa det. 1973, Holótipo.

Conservation status: Good condition; specimen glued on triangle by its abdomen.

Paratype: 1 [unknown sex]. S. Caraça (Engenho), 800m-Minas-XI [1]961, Kloss, Lenko, Martins \& Silva col.; [on red] Buckelater argutus C. Costa det. 1973, Parátipo.

Conservation status: Good condition; specimen glued on triangle by right elytra.

Paratype: 2 [unknown sex]. S. Caraça (Engenho), 1380m-Minas-XI [1]961, Kloss, Lenko, Martins \& Silva col.; [on red] Buckelater argutus C. Costa det. 1973, Parátipo.

Conservation status: Bad condition. Both specimens glued on triangle: one with several appendixes glued on the same triangle; the other with one leg and part of the abdomen glued on the triangle.

Paratype: 2 [unknown sex]. Ponta Grossa, XII-1938, Camargo col.; [on red] Buckelater argutus C. Costa det. 1973, Parátipo.

Conservation status: Bad condition: one totally dissected, glued on triangle under specimen, and 2 slides with mouthparts and genitalia; other glued on triangle with several appendix missing.

Paratype: 3 [unknown sex]. Barueri, SP, Brasil, 21.1955, K. Lenko col., 7270; [on red] Buckelater argutus C. Costa det. 1973, Parátipo.

Conservation status: One slide with one wing; a slide with genitalia; microvial with glycerin with one specimen dissected in bad condition.

Paratype: 2 [unknown sex]. Paraná, Guaraúna, I-1938, J. Pinh. Machado coll.; [on red] Buckelater argutus C. Costa det. 1973, Parátipo.

Conservation status: Both in good condition; glued on triangle by abdome.

Paratype: 2 [unknown sex]. São Paulo, Cantareira, 6181; [on red] Buckelater argutus C. Costa det. 1973, Parátipo.

Conservation status: Idem.

\section{Genus Cardiophorus Eschcholtz, 1829}

Cardiophorus insularis Cobos, 1959: 468

Holotype: 1 q. Coleção M. Alvarenga; [on red] Holótipo; O+; Ilha Fernando de Noronha, Brasil, V.1954, M. Alvarenga; Cardiophorus insularis nov. sp., Holótipo, A. Cobos det. 1958.

Conservation status: Good condition; genitalia glued on white card under specimen.

Paratype: 1 [unknown sex]. [on red] Paratipo; I. Fernando de Noronha, Brasil, V.1954; Cardiophorus insularis nov. sp., Parátipo, A. Cobos det. 1958.

Conservation status: Good condition. 
Cardiorhinus coralinus Golbach, 1983b: 161

Holotype: 1 [unknown sex]. Mury, Nova Friburgo, Rio de Janeiro-Br., 1-31.Jan.1965, Gred \& Guimarães col.; Cardiorhinus coralinus n. sp. det. R. Golbach 1981; [on red] Holótipo.

Conservation status: Good condition. Genitalia and last abdominal segment glued on card under specimen.

\section{Cardiorhinus costae Golbach 1983b: 162}

Holotype: 1 đో. Brasil- Paraná, Ponta Grossa, Camargo col., II.39; đ̊; [on red] Holótipo; C; Cardiorhinus costae n. sp., det. R. Golbach, 1981, Holótipo.

Conservation status: Good condition.

Allotype: 1 ․ Brasil- Paraná, Ponta Grossa, Camargo col., XII.38; ㅇ; [on red] Alótipo; C; Cardiorhinus costae n. sp., det. R. Golbach, 1981, Alótipo.

Conservation status: Good condition.

Paratype: 1 đิ. Paraná, Guararaúna, I-1938; J. Pinha Machado coll.; đ̂; Cardiorhinus costae n. sp., det. R. Golbach, 1981; [yellow] Parátipo.

Conservation status: Good condition.

Cardiorhinus parapullatus Golbach, 1983b: 165

Holotype: 1 đิ. Coleção J. Lane; São Paulo, Pres. Epitácio, J. Lane col., x.54; Pto. Albano, Rio Paraná; C; đే; [on red] Holótipo; Cardiorhinus parapullatus n. sp., det. R. Golbach, 1981.

Conservation status: Good condition; specimen glued on triangle by left elytra.

Paratype: 1 đ̂. Castilho, Marg. esq., R. Paraná, SP, X.1964. Exp. Depto. Zool.; [on yellow]; Cardiorhinus parapullatus $\mathrm{n}$. sp., det. R. Golbach, 1981.

Conservation status: Good condition.

Paratypes: 2 đิ. São Paulo, P. Epitácio Rabello, 10.x.1954; đ̊; Cardiorhinus parapullatus n. sp., det. R. Golbach, 1981. [on yellow] Parátipo.

Conservation status: Good condition.

Cardiorhinus schwarzi Golbach, 1983a: 47

Paratype: 1 ㅇ. 18.505, São Paulo, Ypiranga; ㅇ; [on yellow] PARÁTIPO; Cardiorhinus schwarzi n. sp., det. R. Golbach 1979.

Conservation status: Good condition; left antenna broken.

Cardiorhinus saulensis Chassain, 2011: 209

Paratypes: 1 [unknown sex]. Guyane Française, RN2 PK65 5.10.08, piège vitre SEAG; Doação J. Chassain; [on red] Cardiorhinus saulensis Chassain; [on red] Parátipo.

Conservation status: Good condition; specimen glued by elytra on white card; left antenna glued on the same card.

Paratypes: 1 [unknown sex]. Mgte d. Chevaux, RN2 PK22 Roura; Piège Vitre, 1.xi.2009 SEAG; Doação J. Chassain; [on red] Cardiorhinus saulensis Chassain; [on red] Parátipo.

Conservation status: Good condition, specimen glued on white card. Genitalia glued along in the same card.

Cardiorhinus ypirangus Golbach, 1983b: 168

Holotype: 1 [unknown sex]. Ypiranga; Cardiorhinus ypirangus n. sp., det. R. Golbach 1981; [on red] Holótipo.

Conservation status: Good condition; left antenna missing. Genitalia glued on transparent card under specimen. 
Chalcolepis austerus Casari, 1999: 205

Paratype: 1 [unknown sex]. Costa Rica, San José; Museum Paris, ex Coll., R. Oberthur; Chalcolepis austerus Casari, 1999, S. A. Casari det. 1999; [on red] Paratype.

Conservation status: Good condition.

Paratype: 1 ㅇ. 우 Purula, Vera Paz, Champion; B.C.A Col. III (1). Chalcolepis [illegible] Cand; Chalcolepis austerus Casari, 1999, S. A. Casari det. 1999; [on red] Paratype.

Conservation status: Good condition; genitalia into microvial with glycerin under specimen; left proleg glued on triangle under specimen.

\section{Genus Chalcolepidius Eschscholtz, 1829}

Chalcolepidius albiventris Casari, 2002: 276

Holotype: 1 $\delta$. México, Soterpan (Veracruz) 300m, X.1985 T. Porion; SRGI; Retained SRGI; Chalcolepidius albiventris S. A. Casari. 2002; [on red] Holotype.

Conservation status: Good condition; some antenomers missing on both antennae.

\section{Chalcolepidius serricornis Casari, 2002: 329}

Paratype: 1 ठ․ México, Veracruz, Los Turtlas, 6-IV-87, C. Mayoiga; Coleccion del Instituto de Biologia, UNAM, México, D.F.; Retained UNAM; Chalcolepidius serricornis S. A Casari, 2002; [on red] Paratype; [on red] Paratype.

Conservation status: Good condition; genitalia into microvial with glycerin under specimen.

Paratype: 1 $\delta$. Mexico, Soteapon (Veracruz) $500 \mathrm{~m}$, out 1989, T. Porion cedit; Retained SRGI; SRGI; Chalcolepidius serricornis S. A Casari, 2002; [on red] Paratype; [on red] Paratype.

Conservation status: Good condition; genitalia into microvial with glycerin under specimen.

\section{Chalcolepidius truncuvittatus Casari, 2002: 336}

Holotype: 1 $\delta$. MEX Tamaulipas, Bocotoma area $7 \mathrm{~km}$ SSE Gomez Farias, June 1-4 1982, J. E. Wappes; Chalcolepidius eschcholtzi Cherr. det. E. C. Becker 1988; Retained JEWC; Chalcolepidius truncatipennis S. A Casari, 2002; [on red] Holotype.

Conservation status: Good condition.

\section{Chalcolepidius virgatipennis Casari, 2002: 341}

Paratype: 1 [unknown sex]. Paratype labels: Mex; Museum Paris, Coll. E. FLEUTIAUX; Retained MNHN; Chalcolepidius virgatipennis S. A Casari, 2002; [on red] Paratype; [on red] Paratype.

Conservation status: Good condition; genitalia into microvial with glycerin under specimen.

\section{Genus Cleidecosta Johnson, 2002}

Cleidecosta obscurus (Costa, 1975b): 74

Holotype: 1 đో. Pernambuco, Tapera, 1-932; 468; [on red] Holótipo; Heligmus obscurus C. Costa det. 1975, holótipo.

Conservation status: Good condition; genitalia glued on transparent card under specimen.

Additional notes: The species was originally described in the genus Heligmus Candèze, 1857, a preoccupied name, replaced by Cleidecosta Johnson (2002). 
Paratype: 1 [unknown sex]. Bolivia, Pcia. Ienillo, Buenavista II.1950, A. Martinez leg.; [on red] Holótipo; Heligmus obscurus C. Costa det. 1975, parátipo.

Conservation status: Good condition.

Paratype: 1 [unknown sex ]. Xingu, M. Grosso, BR. IX. 1955, O. VILLA BOAS; MNRJ; [on red] Holótipo; Heligmus obscurus C. Costa det. 1975, parátipo.

Conservation status: Good condition.

Paratype: 1 ㅇ. Guaíra (Sete Quedas)—PR—Brasil, 9-XII.1969, G.R. Kless col.; [on red] Holótipo; Heligmus obscurus C. Costa det. 1975, parátipo.

Conservation status: Good condition; abodmen glued on card under specimen; genitalia glued on transparent card under specimen.

\section{Genus Coctilelater Costa, 1975}

Coctilelater minimus Rosa, 2009: 36

Paratype: 1 [unknown sex]. Brasil, Pará, Serra Norte, N1. Floresta, 31.X a 2. XI.1985; Brasil, Pará, F. F. Ramos; Armadilha Malayse; [on red] Coctilelater minimus Rosa, Paratype; Retido.

Conservation status: Good condition; glued on triangle by mesoventrite.

Coctilelater rosae Chassain, 2009: 55

Paratypes: $1 \hat{\delta}, 4$ [unknown sex]. Guyane frçaise, Bélize; Piége intercept, Novembre 2006[3 specimens] Novembre 2005 [2 specimens], JL Giuglaris; [on red] Paratype; Coctilelater rosae n. sp., J. Chassain, 2007.

Conservation status: Good condition; all specimens mounted on white cards; genitalia of one male glued on the same card.

Paratype: 1 [unknown sex]. Guyane frçaise, JL Giuglaris leg.; Regina, RN2, pk125, 21.X.2007; [on red] Paratype; Coctilelater rosae n. sp., J. Chassain, 2008.

Conservation status: Good condition.

Paratypes: 2 [unknown sex]. Guyane frçaise, Mtgne des Chevauxm RN2 PK22, Roura 27.10.2008 SEAG; Plège intercept. à vitre; [on red] Paratype; Coctilelater rosae n. sp., J. Chassain, 2009.

Conservation status: Good condition.

\section{Genus Ctenicera Latreille, 1829}

Ctenicera comes (Brown, 1939): 48

Paratypes: 1 [unknown sex]. Vancouver, B.C., 31.V.1931, H.B. Leech; [on yellow] Paratype, Ludius comes No. 04421 Brown.

Conservation status: Good condition.

\section{Genus Cyathodera Blanchard, 1846}

\section{Cyathodera auripilosus Costa, 1968: 161}

Holotype: 1 ठ․ Unaí (Faz. Bolívia), MG, Brasil, 22-24.X.1964, Exp. Dep. Zoologia; [on red] Holótipo; [on red] Anoplischius (Cyathodera) auripilosus Costa det. 1968, holótipo.

Conservation status: Good condition; one posterior leg and genitalia glued on transparent cards under specimen.

Additiona notes: The species was described in the genus Anoplischius when Cyathodera was then a subgenus. Johnson (2002) elevated Cyathodera to genus level.

Paratypes: 2 ठิ. Idem, parátipo.

Conservation status: Good condition. 
Paratype: 1 ઈ. Morro da Garça, MG-Brasil, 18-20.X.1964, Exp. Dep. Zoologia; [on red] Anoplischius (Cyathodera) auripilosus Costa det. 1968, parátipo.

Conservation status: Idem.

Paratype: 1 §. Dianópolis, GO, Brasil, 11-14.I.1962, J. Bechyné col.; [on red] Anoplischius (Cyathodera) auripilosus Costa det. 1968, parátipo.

Conservation status: Idem.

\section{Genus Dalopius Eschscholtz, 1829}

Dalopius cognatus Brown, 1934a: 38

Paratype: $1 \delta$. Gravenhuset July, 1932, Ont., G. M. Stirret; [on yellow] Paratype, D. cognatus Brown, No. 3544; Dalopius cognatus.

Conservation status: Good condition; left antenna missing. Specimen glued on white triangle by mesoventrite.

Paratype: 1 ㅇ. Knowlton, Que, 14.6.1928, W.J. Brown; 우, 1 1⁄2; [on yellow] Paratype, D. cognatus Brown, No. 3544.

Conservation status: Idem.

Dalopius fucatus Brown, 1934d: 104

Paratype: 1 ․ Nest Pass., Alta, 9.vi.1930, J. H. Pepper; o⿱; [on yellow] Paratype, D. fucatus Brown, No. 3576.

Conservation status: Good condition. Specimen glued on white triangle by mesoventrite.

Dalopius gentilis Brown, 1934b: 71

Paratype: 1 ㅇ. Go Home Bay, Ont. 6-vii-1932, G.S. Walley; Flat Rock Falls, Go Home R.; + ; [on yellow] Paratype, D. gentilis Brown, No. 3552.

Conservation status: Good condition. Specimen glued on white triangle by mesoventrite.

Dalopius gartrelli Brown, 1934c: 89

Paratype: 1․ Summerland, B.O. 29-vi-1932; [on yellow] Paratype, D. gartrelli Brown, No. 3555.

Conservation status: Good condition. Specimen glued on white triangle by mesoventrite.

Paratype: 1 [unknown sex]. Summerland, B.O. 9-vi-1932; [on yellow] Paratype, D. gartrelli Brown, No. 3555.

Conservation status: Good condition. Glued on white triangle by mesoventrite.

Dalopius gracilis Brown, 1934c: 92

Paratype: 1 + .Berkeley, Calif, April, 1932, H.W. Saylor; [on yellow] Paratype, D. gracilis Brown, No. 3562.

Conservation status: Good condition. Specimen glued on white triangle by mesoventrite.

Dalopius pallidus Brown, 1934c: 87

Paratype: 1 [unknown sex]. Aylmer, Que., 19-vi-1932, W.J. Brown; [on yellow] Paratype, D. pallidus Brown, No.3554.

Conservation status: Good condition. Specimen glued on white triangle by mesoventrite.

Paratype: 1 ․ Aylmer, Que., 30-vi-1932, W. J. Brown; [on yellow] Paratype, D. pallidus Brown, No.3554.

Conservation status: Good condition. Specimen glued on white triangle by mesoventrite. 
Dalopius ignobilis Brown, 1934d: 107

Paratype: 1 +.Fallen Leaf; Lake, vi.24.30; Lake Tahoe, Calif.; A.T. McClay collector; [on yellow] Paratype, $D$. ignobilis Brown, No. 3579.

Conservation status: Good condition. Specimen glued on white triangle by mesoventrite.

Dalopius tristis Brown, 1934d: 105

Paratype: 1 ․ Creston, B.C., 28.iv.1931; G. Stace Smith; ; [ [on yellow] Paratype D. tristis Brown, No. 3577.

Conservation status: Good condition. Specimen glued on white triangle by mesoventrite.

Dalopius vagus Brown, 1934b: 66

Paratype : 1 ․ Aylmer, Que., 17.VI.1932, W.J. Brown; [on yellow] D. vagus Brown, No. 3545.

Conservation status: Good condition. Specimen glued on white triangle by mesoventrite.

Paratype: 1 [unknown sex] Aylmer, Que., 30.vi.1932, W.J. Brown; [on yellow] D. vagus Brown, No. 3545.

Conservation status: Good condition. Specimen glued on white triangle by mesoventrite.

Genus Dicrepidius Eschscholtz, 1829

Dicrepidius brasilianus Casari, 2009: 55

Holotype: 1 ๙ิ. Brasil, Mato Grosso, Nova Mutum, Faz. Buriti, 04/14-II-2002, H.F. Mendes; Elateridae, Coleoptera; Dicrepidius brasilianus SA Casari, 2009 Holotype.

Conservation status: Good condition; genitalia into microvial with glycerin under specimen.

\section{Genus Fulgeochlizus Costa, 1975}

Fulgeochlizus noctivagus Costa, 1991: 567

Holotype: 1 ठ․ Argentina, Peia San Juan, Marayes 13.X.1964, A. Martinez leg.; [on red] Fulgeochlizus noctivagus holótipo, C. Costa det. 1990.

Conservation status: Good condition; genitalia glued on transparent card under specimen.

Paratypes: 7 [unknown sex], $1 \delta$. Argentina, Peia San Juan, Marayes 13.X.1964, A. Martinez leg.; [on red] Fulgeochlizus noctivagus parátipo, C. Costa det. 1990.

Conservation status: Good condition; one specimen with one antenna glued on transparent card under specimen; 1 male with genitalia glued on transparent card under specimens; and 2 specimens with abdomen glued on cards under specimen.

Paratype: $1 \delta$. Argentina, La Rioja, Mascasin I-959; [on red] Fulgeochlizus noctivagus parátipo, C. Costa det. 1990.

Conservation status: Good condition; genitalia glued on transparent card under specimen.

Paratype: $1 \hat{\delta}$. Argentina, La Rioja, Mascasin II-957; [on red] Fulgeochlizus noctivagus parátipo, C. Costa det. 1990; CDA.

Conservation status: Good condition; genitalia glued on transparent card under specimen.

Paratype: 1 [unknown sex]. Argentina, La Rioja, Capital, 6.III-970, Porter-Stange; Entomofauna Subandina; Inst. M. Lillo; [on red] Fulgeochlizus noctivagus parátipo, C. Costa det. 1990; CDA.

Conservation status: Good condition.

Paratype: 1 [unknown sex]. Argentine, La Rioja Prov., Patquia. K.J. Hayward. B.M. 1933-333 Inst.; BM; [on red] Fulgeochlizus noctivagus parátipo, C. Costa det. 1990; CDA.

Conservation status: Good condition.

Paratype: 1 [unknown sex]. Argentina, Catamarca, El Rodeo, 8.28. I. 1959, Col. R. Golbach; Inst. M. Lillo; [on red] Fulgeochlizus noctivagus parátipo, C. Costa det. 1990; CDA.

Conservation status: Good condition. 
Fulgeochlius pullatus Costa, 1991: 571

Paratype: 1 [unknown sex].Paratype labels: Filadelfia, Fer. Col. Chaco, Parag. XI-15-55; Light; J. L. Nickel Collector; Coll. MZSP, São Paulo BR, Ex. Coll. EMEC; [on red] Fulgeochlius pullatus sp n., parátipo, C. Costa det. 1990 .

Conservation status: Good condition.

\section{Genus Lygelater Costa, 1975}

Lygelater succinum Costa, 1980a: 303

Holotype: 1 đ..Bugaba, 800-1500 ft., Champion; B. C. A. Coll. III (1), Pyrophorus fulgidus Ger.; BM; [on red] Lygelater succirous holótipo, C. Costa det. 1979; [on red] holótipo.

Conservation status: Good condition; genitalia and abdomen glued on transparent card under specimen.

Paratype: 1 오. Bugaba, 800-1500 ft., Champion; B. C. A. Coll. III (1), Pyrophorus fulgidus Ger.; BM; [on red] Lygelater succirous parátipo, C. Costa det. 1979; 우.

Conservation status: Good condition.

Lygelater torridus Costa, 1980a: 304

Holotype: 1 đో. Canindé (Rio Gurupi), Pará, Brasil, XII.1964, B. Malkin col.; [on red] Holótipo; [on red] Lygelater torridus holótipo, C. Costa det. 1979.

Conservation status: Good condition; abdomen and genitalia glued on card under specimen.

Paratype: 1 đ̊. Canindé, Rio Gurupi, Pará, Brasil, VI.1963, B. Malkin col.; [on red] Lygelater torridus parátipo, C. Costa det. 1979.

Conservation status: Good condition; abdomen and genitalia glued on card under specimen.

Paratype: 1 ․ Borba, Amazonas, 7.943; Coll. J. Guérin, S. Paulo Brasil, 15968; ㅇ; IB; [on red] Lygelater torridus parátipo, C. Costa det. 1979.

Conservation status: Good condition.

Paratype: 1 q.: Lago Acará, Mb Borba, m/d Rio Madeira, 20.VIII.10.IX.1943, A. Parteo; CS; [on red] Lygelater torridus parátipo, C. Costa det. 1979.

Conservation status: Good condition; abdomen and genitalia glued on card under specimen.

Paratypes: 1 ㅇ, 1 ô. Coleção Campos Seabra; Mangabeira Mocajuba, Para Brasil, II-1953, Orlando M. Rego; 112; [on red] Lygelater torridus parátipo, C. Costa det. 1979.

Conservation status: Good condition; female with abdomen and genitalia glued on card under specimen; male with genitalia into microvial with glycerin.

Paratype: 1 [unknown sex]. Coll. Janson; Ega, Amazons, Bates; BM; 99; [on red] Lygelater torridus parátipo, C. Costa det. 1979.

Conservation status: Good condition.

Paratype: 1 [unknown sex]. Brazil; E.Y. Western Coll., B. M. 1924-176.; BM; 122; [on red] Lygelater torridus parátipo, C. Costa det. 1979.

Conservation status: Good condition.

Paratype: 1 [unknown sex]. Janson coll., ex. Schaum., 1903-130; Bq.; ignitus, F., Cayenne; BM; [on red] Lygelater torridus parátipo, C. Costa det. 1979.

Conservation status: Good condition.

Paratype: 1 [unknown sex]. Parintins, Amazonas, 1.1940; Coll. J. Guérin, S. Paulo Brasil, 9199; IB; ᄋ+; 100; [on red] Lygelater torridus parátipo, C. Costa det. 1979.

Conservation status: Good condition; abdomen and genitalia glued on card under specimen.

Paratype: 1 [unknown sex]. Brazil. Coll. Janson; BM; 121; [on red] Lygelater torridus parátipo, C. Costa det. 1979.

Conservation status: Good condition.

Paratype: 1 [unknown sex]. Cayenne; Coll. Janson., Ex Dejean; 113; Pyrophorus ignitus Fab., Candeze det.; P. ignitus Fab, Cayenne; BM; 95; [on red] Lygelater torridus parátipo, C. Costa det. 1979. 
Conservation status: Good condition.

Paratype: 1 [unknown sex]. Guyane; Collection E. Candèze; Pyrophorus ignitus Fab., Candeze det.; [on red] Lygelater torridus parátipo, C. Costa det. 1979; Coll. MZSP, São Paulo BR, Ex Coll ISNB.

Conservation status: Good condition.

Paratype: 1 [unknown sex]. Cayenne, Coll. Madon; [on red] Lygelater torridus parátipo, C. Costa det. 1979; Coll. MZSP, São Paulo BR, Ex Coll ISNB.

Conservation status: Good condition.

Paratype: 1 [unknown sex]. Mangabeira Mocajuba, Para Brasil, II-1953; CS; Pa; 107; [on red] Lygelater torridus parátipo, C. Costa det. 1979.

Conservation status: Good condition.

Paratype: 1 . Obidos, Amazonas, 5.1929, B. Pohl; Elat. 115; P. ignitus; IB; 115; [on red] Lygelater torridus parátipo, C. Costa det. 1979.

Conservation status: Good condition; female with abdomen and genitalia glued on card under specimen.

Paratype: 1 +. Coleção Campos Seabra; Óbidos, Pará, Brasil, IV-1955, J. Braziline; CS; 114; [on red] Lygelater torridus parátipo, C. Costa det. 1979.

Conservation status: Good condition; genitalia glued on card under specimen.

\section{Genus Globothorax Fleutiaux, 1891}

Globothorax latidens Rosa, 2011: 13

Holotype: 1 J. Cuiabá-MT, Faz. Mutuca, vale do R. Mutuca 4-11.X.2008, interceptação, J. R. M. Rocha, A. L. Silva \& F. H. Gava col.; [on red] Holótipo; [on red] Globothorax latidens Rosa, 2011, Holótipo.

Conservation status: Good condition; glued on triangle by mesosternum.

Paratypes: 261 Oे. 200 idem holotype; 52 idem holotype, collected on 25.X.2008; 9 idem holotype, collected on 29.XI-6.XII.2008.

Conservation status: Idem.

\section{Genus Hapsodrilus Costa, 1975}

Hapsodrilus amydrus Casari-Chen, 1986: 221

Holotype: $1 \delta$. Holotype labels: Costa Rica-MS, 21-25.X.[19]84, Exp. MZSP e IQUSP col.; [on red] Holótipo; [on red] Hapsodrilus amydrus S. A. C-Chen, 1985.

Conservation status: Good condition, genitalia into microvial with glycerin under specimen.

Paratype: 1 ठ. Idem, [on red] Parátipo.

Conservation status: Idem.

\section{Genus Horistonotus Candèze, 1860}

Horistonotus angustifrons Casari, 2011: 3

Holotype: 1 đ. Canindé do S. Fco (SE), (Faz. Brejo), 22.IV.2000 am 1, Tannuzi, L. col. (Malaise); Proj. Xingó, Biodiversidade, Área 5; [on red] Holótipo; Horistonotus angustifrons Casari, 2011.

Conservation status: Good condition.

Paratype: 1 [unknown sex]. Idem; [on red] Parátipo; Horistonotus angustifrons Casari, 2011.

Conservation status: Idem.

Paratype: 2 [unknown sex]. Canindé do S. Fco (SE), (Faz. Brejo),15.IV.2000 am 1, Tannuzi, L. col. (Malaise); Proj. Xingó, Biodiversidade, Área 5; [on red] Parátipo; Horistonotus angustifrons Casari, 2011.

Conservation status: One in good condition; other totally dissected, glued on triangle, with genitalia dissected under specimen into microvial with glycerin. 
Paratype: 1 [unknown sex]. Canindé do S. Fco. (SE), (Faz. Poço Verde), 18.III.2000 (am 1), Ianuzzi, L. col., (Malaise); [on red] Parátipo; Horistonotus angustifrons Casari, 2011.

Conservation status: Good condition.

Horistonotus caatinguensis Casari, 2011: 5

Holotype: 1 ठ . Canindé do S. Fco (SE), (Faz. Poço Verde), 11.III.2000 am 1, Ianuzzi, L. col., (Malaise); Projeto Xingó, Biodiversidade Area 3; [on red] Holotype; Horistonotus caatinguensis Casari, 2011.

Conservation status: Good condition.

Paratype: 1 [unknown sex]. Canindé do S. Fco (SE), (Reserva Chesf), 14.IX.2000 am 3, Ianuzzi, L. col., (Malaise); Projeto Xingó, Biodiversidade Area 1; [on red] Paratype; Horistonotus caatinguensis Casari, 2011. Conservation status: Good condition; specimen glued on triangle by mesoventrite.

Paratype: 1 [unknown sex]. Olho d'água do Casado (AL), (Faz. Talhado), 7.XII.2000 am 4, Ianuzzi, L. col., (Malaise); Projeto Xingó, Biodiversidade Area 6; [on red] Paratype; Horistonotus caatinguensis Casari, 2011. Conservation status: Idem.

Paratype: 1 [unknown sex]. Canindé do S. Fco (SE), (Faz. Poço Verde), 4.III.2000, Ianuzzi, L. col., (Malaise); Projeto Xingó, Biodiversidade Area 4; [on red] Paratype; Horistonotus caatinguensis Casari, 2011.

Conservation status: Good condition. The material was metal coated for SEM and is to the side of the labels. Genitalia are in a microvial with glycerin.

Horistonotus delicatus Casari, 2011: 7

Holotype: 1 đิ. Piranhas (AL), (Faz. Mecejana), 15.IV.2000 am 1, Ianuzzi, L. col., (Malaise); Projeto Xingó Biodiversidade, Área 7; [on red] Holotype; Horistonotus delicatus Casari, 2011.

Conservation status: Good condition; specimen glued on triangle by mesoventrite.

Paratype: 1 [unknown sex]. Canindé do S. Fco. (SE), (Faz. Brejo), 15.IV.2000 am 1, Ianuzzi, L. col., (Malaise); Projeto Xingó Biodiversidade, Área 6; 321; Horistonotus sp., S. Rosa det. 2002; [on red] Paratype; Horistonotus delicatus Casari, 2011.

Conservation status: Idem.

Paratype: 1 [unknown sex]. Canindé do S. Fco. (SE), (Faz. Poço Verde), 22.III.2001 am 5, Ianuzzi, L. col., (Malaise); Projeto Xingó Biodiversidade, Área 5; [on red] Paratype; Horistonotus delicatus Casari, 2011. Conservation status: Idem.

Paratypes: 2 [unknown sex]. Canindé do S. Fco. (SE), (Faz. Poço Verde), 25.III.2001 am 1, Ianuzzi, L. col., (Malaise); Projeto Xingó Biodiversidade, Área 1; [on red] Paratype; Horistonotus delicatus Casari, 2011. Conservation status: Idem.

Paratype: 1 [unknown sex]. Canindé do S. Fco (SE), (Faz. Poço Verde), 22.IV.2000 am 1, Ianuzzi, L. col., (Malaise); Projeto Xingó Biodiversidade, Área 1; [on red] Paratype; Horistonotus delicatus Casari, 2011. Conservation status: Idem.

Paratype: 1 [unknown sex]. Canindé do S. Fco (SE), (Reserva da CHESF), 17.VIII.2000 am 3, Ianuzzi, L. col., (Malaise); Projeto Xingó Biodiversidade, Área 8; [on red] Paratype; Horistonotus delicatus Casari, 2011. Conservation status: Idem.

Paratypes: 2 [unknown sex]. Olho d'agua do Casado (AL), (Faz. Capelinha), 1.VII.2000 am 2, Ianuzzi, L. col., (Malaise); Projeto Xingó Biodiversidade, Área 1; [on red] Paratype; Horistonotus delicatus Casari, 2011. Conservation status: Idem.

Paratype: 1 [unknown sex]. Olho d'agua do Casado (AL), (Faz. Vera Cruz), 8.VI.2000 am 2, Ianuzzi, L. col., (Malaise); 319; Horistonotus sp., S. Rosa det. 2002 ; [on red] Paratype; Horistonotus delicatus Casari, 2011. Conservation status: Idem.

Paratype: 1 [unknown sex]. Piranhas (AL), (Faz. Mecejana), 18.III.2000 am 1, Ianuzzi, L. col., (Malaise); Projeto Xingó Biodiversidade, Área 7; [on red] Paratype; Horistonotus delicatus Casari, 2011. Conservation status: Idem.

Paratype: 1 [unknown sex]. Canindé do S. Fco (SE), (Reserva da CHESF), 22.III.2001 am 5, Ianuzzi, L. col., (Malaise); Projeto Xingó Biodiversidade, Área 8; [on red] Paratype; Horistonotus delicatus Casari, 2011. 
Conservation status: Bad condition; prothorax missing; head glued on triangle; genitalia into microvial with glycerin under specimen.

Additional notes: along with the specimen there is a tube with metal piece with a label 2 .

\section{Horistonotus jaburiensis Casari, 2011: 9}

Holotype: 1 đ̊. Canindé do S. Francisco (SE), (Faz. Jaburú), 24.VIII.2000 am 3, Ianuzzi, L. col. (Malaise); Projeto Xingó Biodiversidade, Área 3; [on red] Holotype; Horistonotus jaburiensis Casari, 2011.

Conservation status: Good condition. Along with the specimen there is a tube with bodypart metalalized with a label 7.

\section{Horistonotus sergipinus Casari, 2011: 14}

Holotype: 1 §. Canindé do S. Francisco (SE), (Faz. Jaburú), 25.III.2000 (am 1), Ianuzzi, L. col. (Malaise); Projeto Xingó Biodiversidade, Área 5; [on red] Holotype; Horistonotus sergipinus Casari, 2011.

Conservation status: Good condition; genitalia into microvial with glycerin under specimen.

\section{Horistonotus tricarinatipennis Casari, 2011: 14}

Holotype: 1 ô. Canindé do S. Francisco (SE), (Faz. Poço Verde), 4.III.2000 (am 1), Ianuzzi, L. col. (Malaise); Projeto Xingó Biodiversidade, Área 1; Horistonotus sp., S. Rosa det. 2002; [on red] Holotype; Horistonotus tricarinatipennis Casari, 2011.

Conservation status: Good condition.

Paratypes: 5 [unknown sex]. Canindé do S. Francisco (SE), (Faz. Poço Verde), 4.III.2000 (am 1), Ianuzzi, L. col. (Malaise); Projeto Xingó Biodiversidade, Área 1; [on red] Paratype; Horistonotus tricarinatipennis Casari, 2011. Conservation status: Idem.

Paratype: 1 [unknown sex]. Idem; Horistonotus sp., S. Rosa det. 2002. Conservation status: Idem.

Paratypes: 4 [unknown sex]. Canindé do S. Francisco (SE), (Faz. Poço Verde), 18.III.2000 (am 1), Ianuzzi, L. col. (Malaise); Projeto Xingó Biodiversidade, Área 1; [on red] Paratype; Horistonotus tricarinatipennis Casari, 2011. Conservation status: Idem.

Paratypes: 4 [unknown sex]. Idem, except 25.III.2000. Conservation status: Idem.

Paratypes: 4 [unknown sex]. Idem, except 08.IV.2000. Conservation status: Idem.

Paratypes: 2 [unknown sex]. Idem, except 22.IV.2000. Conservation status: Idem.

Paratype: 1 [unknown sex]. Olho d'Água do Casado (AL), (Faz. Talhado), 7.XII.2000 am 4, Ianuzzi, L. col. (Malaise); Projeto Xingó Biodiversidade, Área 6; [on red] Paratype; Horistonotus tricarinatipennis Casari, 2011. Conservation status: Idem.

Paratype: 1 [unknown sex]. Olho d'Água do Casado (AL), (São José), 16.XI.2000 am 4, Ianuzzi, L. col. (Solo); Projeto Xingó Biodiversidade, Área 3; [on red] Paratype; Horistonotus tricarinatipennis Casari, 2011. Conservation status: Idem.

Paratype: 1 [unknown sex]. Canindé do S. Francisco (SE), (Faz. Poço Verde), 15.IV.2000 am 1, Ianuzzi, L. col. (Malaise); Projeto Xingó Biodiversidade, Área 3; [on red] Paratype; Horistonotus tricarinatipennis Casari, 2011.

Conservation status: Bad condition; material dissected. Several parts into microvial with glycerin under specimen.

Paratype: 1 [unknown sex]. Canindé do S. Francisco (SE), (Faz. Poço Verde), 18.III.2000 am 1, Ianuzzi, L. col. (Malaise); Projeto Xingó Biodiversidade, Área 3; [on red] Paratype; Horistonotus tricarinatipennis Casari, 2011.

Conservation status: Bad condition; abdomen dissected; genitalia into microvial with glycerin under specimen on a glycerin tube.

Additional notes: Two microvials: one with a metalized specimen (labeled as number 4); another with an unmounted material (labeled as number 11) and with the additional information of locality: Canindé, Faz. Poço Verde, 11.III.200, Area 1. 
Horistonotus xingoensis Casari, 2011: 16

Holotype: 1 ठో. Canindé Canindé do S. Francisco (SE), (Faz. Poço Verde), 25.III.2000 am 1, Ianuzzi, L. col. (Malaise); Projeto Xingó Biodiversidade, Área 3; Horistonotus sp., S. Rosa det. 2002; [on red] Paratype; Horistonotus xingoensis Casari, 2011.

Conservation status: Good condition; genitalia into microvial with glycerin under specimen.

Paratype: 1 [unknown sex].: Canindé do S. Francisco (SE), (Faz. Poço Verde), 15.IV.2000 am 1, Ianuzzi, L. col.

(Malaise); Horistonotus sp., S. Rosa det. 2002; [on red] Paratype; Horistonotus xingoensis Casari, 2011.

Conservation status: Good condition.

Paratype: 1 [unknown sex]. Olho d'Água do Casado (AL), (Faz. Talhado), 30.XI.2000 am 4; Ianuzzi, L. col.

(Malaise); Projeto Xingó Biodiversidade, Área 6; [on red] Paratype; Horistonotus xingoensis Casari, 2011.

Conservation status: Good condition.

Horistonotus nordestinus Casari, 2011: 9

Holotype: 1 ð. Canindé do S. Fco (SE), (Faz. Poço Verde), 15.IV.2000 am1, Ianuzzi, L. col. (Malaise); Projeto Xingó Biodiversidade, Área 1; [on red] Holotype; Horistonotus nordestinus Casari, 2011. Conservation status: Good condition.

Paratypes: 4 [unknown sex]. Canindé do S. Fco (SE), (Res. Chesf) 17.VIII.2000 am3, Ianuzzi, L. col. (Malaise); Projeto Xingó Biodiversidade, Área 1; [on red] Paratype; Horistonotus nordestinus Casari, 2011.

Conservation status: Good condition; specimens glued on triangles by mesoventrite.

Paratypes: 3 [unknown sex]. Canindé do S. Fco (SE), (Faz. Jaburú) 17.VIII.2000 am3, Ianuzzi, L. col. (Malaise);

Projeto Xingó Biodiversidade, Área 3; [on red] Paratype; Horistonotus nordestinus Casari, 2011.

Conservation status: Idem.

Paratypes: 6 [unknown sex]. Idem, except for the date of collection 14.IX.2000. Conservation status: Good condition.

Paratypes: 2 [unknown sex]. Canindé do S. Fco (SE), (Res. Chesf) 1.VI.2000 am2, Ianuzzi, L. col. (Malaise);

Projeto Xingó Biodiversidade, Área 8; [on red] Paratype; Horistonotus nordestinus Casari, 2011.

Conservation status: Good condition; specimens glued on triangles by mesoventrite.

Paratypes: 2 [unknown sex]. Canindé do S. Fco (SE), (Res. Chesf) 1.III.2000 am5, Ianuzzi, L. col. (Malaise);

Projeto Xingó Biodiversidade, Área 8; [on red] Paratype; Horistonotus nordestinus Casari, 2011. Conservation status: Idem.

Paratypes: 3 [unknown sex]. Olho d'Água do Casado (SE), (Faz. Capelinha) 11.V.2000 am2, Ianuzzi, L. col.

(Malaise); Projeto Xingó Biodiversidade, Área 1; [on red] Paratype; Horistonotus nordestinus Casari, 2011. Conservation status: Good condition; specimens glued on triangles by mesoventrite.

Paratypes: 3 [unknown sex]. Olho d'Água do Casado (SE), (Faz. São José) 23.XI.2000 am4, Ianuzzi, L. col.

(Malaise); Projeto Xingó Biodiversidade, Área 4; [on red] Paratype; Horistonotus nordestinus Casari, 2011. Conservation status: Idem.

Paratypes: 3 [unknown sex]. Olho d'Água do Casado (SE), (Faz. Talhado) 30.XI.2000 am4, Ianuzzi, L. col.

(Malaise); Projeto Xingó Biodiversidade, Área 6; [on red] Paratype; Horistonotus nordestinus Casari, 2011. Conservation status: Idem.

Paratypes: 3 [unknown sex]. Idem, 26.X.2000, Área 5. Conservation status: Idem.

Paratypes: 3 [unknown sex]. Piranhas (AL), (Faz. Mecejana), 18.III.2000 (am 1), Ianuzzi, L. col. (Malaise.);

Projeto Xingó Biodiversidade, Área 7; [on red] Paratype; Horistonotus nordestinus Casari, 2011.

Conservation status: Good condition; specimens glued on triangles by mesoventrite.

Paratype: 1 [unknown sex].: Idem, except for the date of collection 25.III.2000. Conservation status: Idem.

Paratypes: 3 [unknown sex]. Olho d’Água do Casado (SE), (Faz. Talhado) 7.XI.2000 am4, Ianuzzi, L. col. (Malaise); Projeto Xingó Biodiversidade, Área 6; [on red] Paratype; Horistonotus nordestinus Casari, 2011. Conservation status: Idem.

Paratypes: 2 [unknown sex]. Piranhas (AL), (Faz. Mecejana), 08.IV.2000 am1, Ianuzzi, L. col. (Malaise). Projeto Xingó Biodiversidade, Área 7; [on red] Paratype; Horistonotus nordestinus Casari, 2011. 
Conservation status: Idem.

Paratypes: 5 [unknown sex]. Idem, except 15.IV.2000.

Conservation status: Idem.

Paratype: 1 [unknown sex]. Olho d'agua do Casado (AL), (Faz. Capelinha), 25.V.2000 am 2, Ianuzzi, L. col.

(Malaise); Projeto Xingó Biodiversidade, Área 1; [on red] Paratype; Horistonotus nordestinus Casari, 2011.

Conservation status: Idem.

Paratype: 1 [unknown sex]. Olho d'agua do Casado (AL), (Faz. São José, 31.IX.2000 am 42, Ianuzzi, L. col. (Solo); Projeto Xingó Biodiversidade, Área 4; [on red] Paratype; Horistonotus nordestinus Casari, 2011. Horistonotus sp. S. Rosa det. 2002.

Conservation status: Idem.

Paratypes: 3 [unknown sex]. Canindé do S. Fco (SE), (Faz. Brejo), 4.III.2000 am 1, Ianuzzi, L. col. ( Malaise) Projeto Xingó Biodiversidade, Área 5; [on red] Paratype; Horistonotus nordestinus Casari, 2011.

Conservation status: Idem.

Paratype: 1 [unknown sex]. Canindé do S. Fco. (SE), (Reserva CHESF), 28.IX.2000 am 3, Ianuzzi, L. col. (solo);

Projeto Xingó Biodiversidade, Área 1; [on red] Paratype; Horistonotus nordestinus Casari, 2011. Conservation status: Idem.

Paratypes: 3 [unknown sex]. Piranhas (AL), (Faz. Mecejana), 22.IV.2000 am1, Ianuzzi, L. col. (Malaise); Projeto Xingó Biodiversidade, Área 7; [on red] Paratype; Horistonotus nordestinus Casari, 2011. Conservation status: Idem.

Paratypes: 3 [unknown sex]. Canindé do S. Fco (SE), (Faz. Jaburú), 21.IX.2000 am 3, Ianuzzi, L. col. (Malaise); Projeto Xingó Biodiversidade, Área 3; [on red] Paratype; Horistonotus nordestinus Casari, 2011.

Conservation status: Idem.

Paratypes: 4 [unknown sex]. Canindé do S. Fco (SE), (Faz. Miramar), 08.II.2001 am 5, Ianuzzi, L. col. (3 Malaise,

1 solo); Projeto Xingó Biodiversidade, Área 5; [on red] Paratype; Horistonotus nordestinus Casari, 2011.

Conservation status: Idem.

Paratypes: 6 [unknown sex]. Canindé do S. Fco (SE), (Faz. Miramar), 01.III.2001 am 5, Ianuzzi, L. col. (3 Malaise, 3 solo); Projeto Xingó Biodiversidade, Área 5; [on red] Paratype; Horistonotus nordestinus Casari, 2011. Conservation status: Idem.

Paratypes: 2 [unknown sex]. Canindé do S. Fco (SE), (Faz. Poço Verde), 04.III.200 am 1, Ianuzzi, L. col. (Malaise); Projeto Xingó Biodiversidade, Área 5; [on red] Paratype; Horistonotus nordestinus Casari, 2011. Conservation status: Idem.

Paratype: 1 [unknown sex]. Canindé do S. Fco (SE), (Faz. Esplanada), 28.IX.200 am 3, Ianuzzi, L. col. (Malaise);

Projeto Xingó Biodiversidade, Área 5; [on red] Paratype; Horistonotus nordestinus Casari, 2011. Conservation status: Idem.

Paratypes: 2 [unknown sex]. Canindé do S. Fco (SE), (Faz. Miramar), 15.III.2001 am 5, Ianuzzi, L. col. (solo); Projeto Xingó Biodiversidade, Área 5; [on red] Paratype; Horistonotus nordestinus Casari, 2011. Conservation status: Idem.

Paratype: 1 [unknown sex]. Olho d'agua do Cassado (AL), (Faz. Vera Cruz), 29.VI.2000 am 2, Ianuzzi, L. col.

(Malaise); Projeto Xingó Biodiversidade, Área 5; [on red] Paratype; Horistonotus nordestinus Casari, 2011. Conservation status: Idem.

Paratypes: 4 [unknown sex]. Canindé do S. Fco (SE), (Faz. Poço Verde), 11.III.2000 am 1, Ianuzzi, L. col.

(Malaise); Projeto Xingó Biodiversidade, Área 1; [on red] Paratype; Horistonotus nordestinus Casari, 2011. Conservation status: Idem.

Paratypes: 4 [unknown sex]. Idem, except 4.III.2000. Conservation status: Idem.

Paratypes: 4 [unknown sex]. Canindé do S. Fco (SE), (Faz. Miramar), 22.II.2001 am 5, Ianuzzi, L. col. (3 Malaise,

1 Solo); Projeto Xingó Biodiversidade, Área 5; [on red] Paratype; Horistonotus nordestinus Casari, 2011. Conservation status: Idem.

Paratypes: 3 [unknown sex]. Canindé do S. Fco (SE), (Faz. Poço Verde), 18.II.2000 am 1, Ianuzzi, L. col. (Malaise); Projeto Xingó Biodiversidade, Área 3; [on red] Paratype; Horistonotus nordestinus Casari, 2011. Conservation status: Idem.

Paratypes: 3 [unknown sex]. Idem, 11.III.2000. 
Conservation status: Idem.

Paratypes: 3 [unknown sex]. Canindé do S. Fco (SE), (Faz. Poço Verde), 25.III.2000 am 1, Ianuzzi, L. col. (Malaise); Projeto Xingó Biodiversidade, Área 3; [on red] Paratype; Horistonotus nordestinus Casari, 2011.

Conservation status: Idem.

Paratype: 1 [unknown sex]. Idem, (Faz. Porto Belo), 17.VII.2000, am 3.

Conservation status: Idem.

Paratypes: 3 [unknown sex]. Idem, (Faz. Poço Verde), 22.IV.2000, am 1. Conservation status: Idem.

Paratypes: 2 [unknown sex]. Canindé do S. Fco (SE), (Faz. Poço Verde), 08.IV.2000 am 1, Ianuzzi, L. col. (Malaise); Projeto Xingó Biodiversidade, Área 3; [on red] Paratype; Horistonotus nordestinus Casari, 2011. Conservation status: Idem.

\section{Genus Hypnoidus Stephens, 1830}

Hypnoidus beckeri Stibick, 1980: 254

Paratypes: 1 [unknown sex]. [on a yellow circle] Paratype; India: Bengal, Darjeeling Hills, Sandakphu, $12.000 \mathrm{ft}$. 5.v.1934, H.G. Champion; Paratype, Hypnoidus beckeri Stibick.

Conservation status: Good condition; glued on card.

\section{Genus Hypolithus Eschcholtz, 1829}

Hypolithus nepalensis Ohira \& Becker, 1973: 74

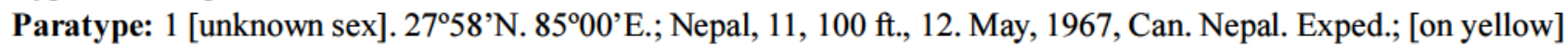
Paratype, CNC No. 13770, Hypolithus nepalensis Ohira \& Becker.

Conservation status: Good condition.

\section{Genus Hypsiophthalmus Latreille, 1834}

\section{Hypsiophthalmus charops Costa, 1978: 267}

Paratype: 1 ठิ. Petrop. Bras.; F. Sabliberg; 1208; Mus. Zool. Helsinski n.o., 1578; [on red] paratipo; [on red] Hypsiophthalmus charops C. Costa det. 1978.

Conservation status: Good condition; abdomen glued on card under specimen; genitalia glued on transparent card under specimen.

Paratype: 1 [unknown sex]. Petrópolis, Feb. 1857, H. Clark; Coll. Janson; BM; [on red] Paratipo; [on red] Hypsiophthalmus charops C. Costa det. 1978.

Conservation status: Good condition; glued on card.

Paratype: 1 [unknown sex]. Fry. Riorani; Fry. Coll. 1905.100; BM; [on red] Paratipo; [on red] Hypsiophthalmus charops C. Costa det. 1978.

Conservation status: Good condition.

Paratype: 1 [unknown sex]. Candéze; 58; Pyrearinus Esch.; Mus. Zool. Helsinki N.o 15579; [on red] Paratipo; [on red] Hypsiophthalmus charops C. Costa det. 1978.

Conservation status: Good condition.

Paratype: 1 [unknown sex]. Petrop. Brs.; F. Sahlb.; 279.; Mus. Zool. Helsinki N.o 15380; [on red] Paratipo; [on red] Hypsiophthalmus charops C. Costa det. 1978.

Conservation status: Good condition.

Paratype: 1 đิ. Monterides; Collection, E. Candéze; Pyrophorus rariesche [ilegible] det. E. Candéze; Coll. MZSP, Sao Paulo BR, Ex. Coll. ISNB; [on red] Paratipo; [on red] Hypsiophthalmus charops C. Costa det. 1978.

Conservation status: Good condition; abdomen glued on card under specimen; genitalia glued on transparent card under specimen. 
Paratype: 1 ठิ. Brasilia; F. Sahlberg; 1207; Mus. Zool. Helsinki N.o 15581; [on red] Paratipo; [on red] Hypsiophthalmus charops C. Costa det. 1978.

Conservation status: Good condition.

Paratype: 1 [unknown sex]. Brasilia; CNHM, 1955, Karl Branesik Collin.; ex. Eduard Knirsch; CNHM; [on red] Paratipo; [on red] Hypsiophthalmus charops C. Costa det. 1978.

Conservation status: Good condition; abdomen glued on card under specimen.

Paratype: 1 [unknown sex]. Specimen orig. without labels in colla but associated with specimen bearing attached data; Brasilia; CNHM, 1955, Karl Breansik Colln., ex. Eduard Knirsc; CNHM; [on red] Paratipo; [on red] Hypsiophthalmus charops C. Costa det. 1978.

Conservation status: Good condition.

Hypsiophthalmus luscius Costa, 1979: 271

Holotype: 1 đ. V. Caraguatá P. Bristal, S. Paulo-Capital, 10.X.1965, Brasil, B.M. Dias-col.; [on red] Hypsiohthalmus luscius sp. nov., holótipo, C. Costa det. 1979; [on red] Holótipo.

Conservation status: Good condition; right antenna missing; abdomen glued on card under specimen; genitalia and last abdominal ventrites glued on transparent card under specimen.

Paratypes: 2 [unknown sex]. 46 ol; [on red] Hypsiophthalmus luscius sp. nov., parátipo, C. Costa det. 1979; [on red] Parátipo; F.

Conservation status: Good condition.

Paratype: 1 [unknown sex]. Ypiranga; [on red] Hypsiophthalmus luscius sp. nov., parátipo, C. Costa det. 1979.

Conservation status: Good condition. Abdomen glued on card under specimen.

Paratype: 1 [unknown sex]. 1969, Jundiahy, E. S. Paulo; [on red] Hypsiophthalmus luscius sp. nov., parátipo, C. Costa det. 1979.

Conservation status: Good condition.

Paratype: 1 [unknown sex]. 49. São Paulo, Capital; F; [on red] Hypsiophthalmus luscius sp. nov., parátipo, C. Costa det. 1979.

Conservation status: Good condition; right antenna missing.

Paratype: $1 \delta^{\lambda}$.Casa Grande, S. Paulo, 3.937; Coll. J. Guérin, S. Paulo, Brasil, 9172; Pyrophorus ranimus Esch., J. Guerin. det. 1942; IB; [on red] Hypsiophthalmus luscius sp. nov., parátipo, C. Costa det. 1979.

Conservation status: Good condition; genitalia and left antenna glued on transparent card under specimen.

Paratype: 1 ठิ. Pyrophorus; Hypsiophthalmus microspilus (Germar, 1841), C. Costa det. 1972; [on red] Hypsiophthalmus luscius sp. nov., parátipo, C. Costa det. 1979; IB.

Conservation status: Good condition; genitalia glued on transparent card under specimen.

\section{Genus Ignelater Costa, 1975}

Ignelater glaesum Costa, 1980b: 159

Paratypes: 1 ठึ, 2 [unknown sex]. 17.iv-26,viii, 1938. Oxf. Un.Cayman Is, Biol. Exped., coll; by C. B. Lewis, G. H. Thompson; 20. V.1929, Cayman Brac, West end of, Cotton-tree, Land. Light trap B.; Pres. Hope, Dept. Oxford, B. M. 1967-147.; BM; [on red] Ignelater glaesum parátipo, C. Costa det. 1979.

Conservation status: Good condition; one specimen with abdomen glued on card under specimen; one specimen with genitalia glued on transparent card.

Ignelater brunneus Costa, 1980b: 158

Paratype: 1 [unknown sex]. Cuba, 1936, Darlington Collector; Mts. N. of Imias eastern Oriente, July 25-28-1936, 8000-4000 ft.; MCZ; [on red] Ignelater brunneus parátipo, C. Costa det. 1979.

Conservation status: Good condition.

Paratype: 1 9. Cuba, 1936, Darlington Collector; Mts. N. of Imias eastern Oriente, July 25-28-1936, 8000-4000 ft.; MCZ; [on red] Ignelater brunneus parátipo, C. Costa det. 1979.

Conservation status: Good condition; genitalia glued on transparent card under specimen. 
Ignelater inaguensis Rosa, 2010: 445

Paratype: $1 \delta$. Blacklight trap in mature mangrove forest; Bahamas: Great Inagua north coast road N21.10813, W73.60196 13-VII-2007; Thomas, Turnbow \& Smith; [on red] Ignelater inaguensis paratype, Rosa, 2010.

Conservation status: Good condition; genitalia in microvial with glycerin under specimen.

\section{Genus Megapenthes Kiesenwetter, 1858}

\section{Megapenthes ibitiensis Navajas, 1944: 71}

Holotype: 1 đे. [on red] Holótipo; Monte Alegre, Fazenda Sta. Maria,Alt. 1.100 mts., 24-30.XI.1942, F. Lane col.; Megapenthes ibitiensis Navajas, 1944, Nav. det. 19; L45; L117; 105.752.

Conservation status: Good condition.

\section{Megapenthes apacheorum Becker, 1971: 157}

Paratype: 1 [unknown sex]. Madera Cn., Sta. Rita, Mts. Ariz. Jul 7, 1961. P. H. Johnson, U.V. lt. trp; [on yellow] Paratype, Megapenthes apacheorum Becker, CNC No. 10899.

Conservation status: Good condition; specimen glued on triangle by right elytra.

Megapenthes texanus Becker, 1971: 160

Paratype: 1 [unknown sex]. TEXAS, Chino Mts., Juniper Canyon, VII-24-1923, 266, F.M. Gaige; [on yellow] Paratype CNC No. 10900, Megapenthes texanus Becker.

Conservation status: Good condition.

\section{Subgenus Xanthopenthes Fleutiaux, 1929}

Megapenthes (Xanthopenthes) almeidai Navajas, 1946: 14

Holotype: 1 [unknown sex]. [on red] Holótipo; Instituto Oswaldo Cruz, Zona da N.O.B, Salobra, 18-29.x. [19]38; Megapenthes (Xanthopenthes) almeidai Navajas, 1945; 105.755; L46; L115; L114.

Conservation status: Good condition.

\section{Genus Meroplinthus Candèze, 1891}

Meroplintus ambrosius Costa, 1975b: 75

Holotype: 1 đో. [on red] Holótipo; Piracicaba, S.P., Ex. col. Ent., E. S. Agu, L. Queiroz, Col. I. Biológico; XL; [on red] Meroplintus ambrosia C. Costa det. 1975, holótipo.

Conservation status: Good condition; left antenna glued on card under specimen.

Paratype: 1 + . Jatahy, Etat de Goyaz, Ch Pujol 1895-96; Muséum Paris, 1952, Coll. R. Oberthur; MNHNP; [on red] Meroplintus ambrosia C. Costa det. 1975, paratipo.

Conservation status: Good condition. Mouthparts and genitalia glued on transparent cards under specimen. Paratype: $1 \delta$. Brésil, Prov. Matto Grosso, P. Germáin, 1886; Muséum Paris, 1952, Coll. R. Oberthur; MNHNP; [on red] Meroplintus ambrosia C. Costa det. 1975, paratipo.

Conservation status: Good condition. Genitália glued on transparent cards under specimen.

\section{Meroplinthus decorus Costa, 1975b: 76}

Holotype: 1 $\delta$. [on red] Holótipo; Sayago, Montevideo, 25-I-1955, A. Silveira; f22; IB; [on red] Meroplinthus decorus C. Costa det. 1975, holótipo.

Conservation status: Good condition.

Paratype: 1 [unknown sex]. Donacion, Tombaxini, 1949; f21; IB; [on red] Meroplinthus decorus C. Costa det. 1975, paratipo. 
Conservation status: Good condition.

Paratype: 1 9. Rio Grande, 85-4; BM; [on red] Meroplinthus decorus C. Costa det. 1975, holótipo.

Conservation status: Good condition. Genitalia glued on transparent card under specimen.

Paratype: 1 [unknown sex]. Pelotas, II. 934, Irmas Figueiredo leg.; BM; [on red] Meroplinthus decorus C. Costa det. 1975, holótipo.

Conservation status: Good condition. Both antennae broken, one glued on card under specimen.

Paratype: 1 [unknown sex]. Saimgo, 1948; f23; IB; [on red] Meroplinthus decorus C. Costa det. 1975, holótipo.

Conservation status: Good condition; left antenna broken.

Genus Metapyrophorus Rosa \& Costa, 2009

Metapyrophorus pharolim Rosa \& Costa, 2009: 45

Holotype: 1 $\delta$. Caparo Trinidad, A. Heynes, Berlin-Wilm; n. sp. Collection Fleutiaux; MNHNP; [on red] Metapyrophorus pharolim Rosa \& Costa, 2009, Holótipo; [on red] Holótipo.

Conservation status: Good condition; genitalia and abdomen glued on a card under specimen.

Paratypes: 2 $\delta$. Idem, parátipo.

Conservation status: Idem; one specimen with abdomen glued on card under specimen.

Paratype: 1 ठ․ St. Anns. Port of Spain, Trinidad, June. 28. 1957, M. Nieves; AMNH; [on red] Metapyrophorus pharolim Rosa \& Costa, 2009, Holótipo; [on red] Holótipo.

Conservation status: Good condition; genitalia into microvial with glycerinunder specimen.

Paratypes: $1 \hat{\delta}, 1$ [unknown sex]. Venezuela, Inst. Zool. Agrícola, Fac. Agronomia, Univ. Central; J. Salgado, L. Rodriguez. V.; Venezuela-Monagas, Jasepin, 5-VI-1967; Venezuela; [on red] Paratype; [on red] Metapyrophorus pharolim Rosa \& Costa, 2009, Parátipo.

Conservation status: Good condition; one specimen with abdomen glued on a card under specimen; other specimen with head and abdomen glued on card under specimen and with genitalia into microvial with glycerin.

Paratype: 1 [unknown sex]. Pie del Cerro, Morrocoy, T.F.A, 31-III-58; talvez + , estava sem a genitalia; MIS; [on red] Paratype; [on red] Metapyrophorus pharolim Rosa \& Costa, 2009, Parátipo.

Conservation status: Good condition; abdomen glued on card under specimen.

Additional note: translated from Portuguese: "maybe + , [the specimen was] without the genitalia".

\section{Genus Neoarhaphes Costa, 1966}

Neoarhaphes brasiliensis Costa, 1966: 264

Holotype: 1 đิ. Buritís, (Ribeirão Confins), MG-29-31.X.1964, Exp. Dep. Zool.; [on red] Neoarhaphes

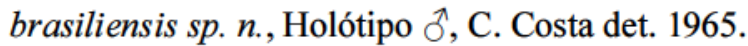

Conservation status: Good condition; abdomen glued on transparent card under specimen.

Allotype: 1 ․ Idem.

Conservation status: Idem.

Paratypes: 2 +, 15 ठ․ Buritís, (Ribeirão Confins), MG-29-31.X.1964, Exp. Dep. Zool.; [on red] Neoarhaphes brasiliensis sp. n., C. Costa det. 1965.

Conservation status: Good condition. Two females with abdomen dissected and glued on transparent card under specimens.

\section{Genus Noxlumenes Costa, 1975}

Noxlumenes bardus Costa, 1975a: 87

Holotype: 1 đ. Las hajas meuquén Argentina, 16.1.1967, S. Martin y Monné; M; [on red] Noxlumens bardus holótipo $\widehat{o}$, C. Costa det. 1975.

Conservation status: Good condition; genitalia and abdomen glued on a card under specimen. 
Paratype: 1 đ. Idem, parátipo.

Conservation status: Idem.

Paratypes: 2 đ. Argentina-Neuquén, Zapala, 23-III-1964, A. Mesa, R. Sanduleki; M; [on red] Noxlumens bardus holótipo $\widehat{\text { Oै }}$, C. Costa det. 1975.

Conservation status: Good condition; one specimen with genitalia into microvial with glycerin, and one with one leg glued on card under it.

\section{Genus Opselater Costa, 1975}

Opselater costae Rosa, 2004: 208

Holotype: 1 đ. Linhares, ES Brasil, XI.1972, P. C. Elias; [on red] Holótipo; [on red] Opsolater costae sp. n., holótipo. S. Rosa det. 2002

Conservation status: Good condition; genitalia into microvial with glycerin under specimen.

Paratypes: 58 [unknown sex]. Linhares, ES Brasil,1972, P. C. Elias; [on red] Parátipo; [on red] Opsolater costae sp. n., parátipo. S. Rosa det. 2002. [ 43 specimens collected on XI.1972; 10 on XII.1972; 3 on X.1972; 2 on I.1972].

Conservation status: Good condition.

Paratype: 1 [unknown sex]. Parque Sooretama, Linhares, E. Santo, Brasil XI.1960, D. Zajciw leg.; [on red] Parátipo; [on red] Opsolater costae sp. n., parátipo. S. Rosa det. 2002.

Conservation status: Good condition.

Paratype: 1 [unknown sex]. Linhares Espirito Santo, Brasil, X.1969, M. Morais col.; [on red] Parátipo; [on red] Opsolater costae sp. n., parátipo. S. Rosa det. 2002.

Conservation status: Good condition.

Paratype: 1 [unknown sex]. Brasil—E. Santo, Linhares, 30.XI.1974, C. S. Carbonell; [on red] Parátipo; [on red] Opsolater costae sp. n., parátipo. S. Rosa det. 2002.

Conservation status: Good condition.

Paratype: 1 [unknown sex]. Coleção Campos Seabra; Linhares, Esp. Santo Brasil, IX.1970, B. Silva; [on red] Parátipo; [on red] Opsolater costae sp. n., parátipo. S. Rosa det. 2002.

Conservation status: Good condition.

Paratype: 1 [unknown sex]. Colatina, E. Santo Brasil, XII.1970, A. Sila; [on red] Parátipo; [on red] Opsolater costae sp. n., parátipo. S. Rosa det. 2002.

Conservation status: Good condition.

Paratype: 1 [unknown sex]. Brasil-ES, Linhares, 18-IX-90, J.S.Santos col.; [on red] Parátipo; [on red] Opsolater costae sp. n., parátipo. S. Rosa det. 2002.

Conservation status: Good condition; genitalia into microvial with glycerin under specimen.

Paratype: 1 [unknown sex]. Idem, 5V.87.

Conservation status: Good condition.

Paratype: 1 [unknown sex]. Idem, 4.III.88.

Conservation status: Idem; genitalia into microvial with glycerin under specimen.

Paratype: 1 [unknown sex]. Idem, 22I.88.

Conservation status: Good condition.

\section{Genus Paranoplischius Casari, 2013}

\section{Paranoplischius nigellus Casari, 2013: 146}

Holotype: 1 $\delta$. Brasil, Santaremsinho, Mun. de ITAITUBA, Rio Tapajós = Pará, IV-[19]61; [on red] Holotype; Paranoplischius nigellus Casari, 2013.

Conservation status: Good condition.

Paratype: 1 [unknown sex]. Brasil: AM, Benj. Constat, Rio Javari, XI.1960, Dirings; [on red] Paratype; Paranoplischius nigellus Casari, 2013.

Conservation status: Good condition; genitalia into a microvial with glycerin under specimen. 
Paratype: 2 [unknown sex]. Brasil: AM, Benj. Constat, Rio Javari, Dirings; [on red] Paratype; Paranoplischius nigellus Casari, 2013.

Conservation status: Good condition.

Paranoplischius modestus Casari, 2013: 144

Holotype: 1 đ̊. Dianópolis, GO, Brasil, 11-14.I.1962, J. Bechyné col.; [on red] Holotype; Paranoplischius modestus Casari, 2013.

Conservation status: Good condition. [on red] Paratype.

Paratype: 1 [unknown sex]. Idem.

Conservation status: Good condition; glued on triangle by elytra.

Paratype: 1 [unknown sex]. Brasil, PA, Itaituba, II.1961, Dirings; [on red] Paratype; Paranoplischius modestus Casari, 2013.

Conservation status: Idem

Paratypes: 2 [unknown sex]. M. Grono, S. Domingos, X.49; [on red] Paratype; Paranoplischius modestus Casari, 2013.

Conservation status: Good condition; one glued on triangle by elytra.

Paratype: 1 [unknown sex]. Paraiba, Areia, VII.955, Pe. Pereira; [on red] Paratype; Paranoplischius modestus Casari, 2013.

Conservation status: Good condition.

Paratype: 1 [unknown sex]. Brasil, Pará. Benevides F.Morelandia, 6.II.1987; [on red] Paratype; Paranoplischius modestus Casari, 2013.

Conservation status: Good condition.

Paratype: 1 [unknown sex]. PA, Peixe Boi, 14.IV.1977; Brasil Pará, WL Overal; ; [on red] Paratype; Paranoplischius modestus Casari, 2013.

Conservation status: Good condition; genitalia into a microvial with glycerin under specimen.

\section{Genus Paratractosomus Casari, 2013}

Paratractosomus sulciventris Casari, 2013: 148

Paratype: 1 [unknown sex]. Bolivia, Santa Cruz, Florida, 4km N Bermejo, 29.X.2011, Coll: Skillman \& Wappes; Refúgio los Volcanes, FWSC Loan, Feb. 2012; Paratractosomus sulciventris Casari, 2013 Paratype.

Conservation status: Good condition.

Paratype: 1 [unknown sex]. Bolivia, Santa Cruz, Florida, 4km N Bermejo, 25-29 October 2011, Coll: Skillman \& Wappes; Refúgio los Volcanes $18^{\circ} 06^{\prime} \mathrm{S}$ 633' W, 1000-1200 meters; Paratractosomus sulciventris Casari, 2013 Paratype.

Conservation status: Good condition, genitalia into a microvial with glycerin under specimen.

\section{Genus Physodactylus Fischer, 1823}

Physodactylus fleutiauxi Chassain, 2005: 68

Paratype: 1 ô. Brésil (Mato Grosso), $14^{\circ} 15^{\prime} 50.80^{\prime \prime} \mathrm{S}, 59^{\circ} 14^{\prime} 02.05^{\prime \prime} \mathrm{W}$, Chapada dos Parecis; $30 \mathrm{~km}$ Nord d'Uirapuru, Usine Alcomat, 1 au 15-XII-2001, A. Foucart leg.; [on red] Paratype; Physodatctylus fleutiauxi n. sp., J. Chassain det. $04 ;$ P. fleutiauxi.

Conservation status: Good condition; glued on card. Genitalia glued on card under specimen.

\section{Pseudanostirus Dolin, 1964}

Pseudanostirus propolus columbianus (Brown, 1935b): 185 
Paratype: 1 [unknown sex]. Vernon, B.C., V.I.1924. R. Hoppiza; [on yellow] L. propola columbianus, No. 3903 Brown.

Conservation status: Good condition.

Additional notes: Described as a subspecies it was elevated to species by Brooks (1960), then returned to subspecies rank by Lane (1971). Gurjeva (1989) transferred the nominal species to Pseudanostirus.

\section{Genus Ptesimopsia Costa, 1975}

Ptesimopsia brunnea Costa, 1975b: 57

Holotype: 1 ठో. Est. Biol. Boraceia, Salesópolis, SP, I.1950, E. X. Rabello col.; [on red] Ptesimopsia brunnea sp. nov., holótipo, C. Costa det. 1975.

Conservation status: Good condition; left antenna missing; genitalia and last abdominal segments glued on transparent card under specimen.

\section{Ptesimopsia elongata Costa, 1975b: 58}

Holotype: 1 đ. Eug. Lefevre 1200 m., Pindamonhangaba, S.P., 21.XII.1962; Exp. Dep. Zool.; [on red] Ptesimopsia elongata C.Costa det. 1975, holotype $\delta^{\star}$.

Paratype: $1 \delta$. Idem, paratype.

Conservation status: Good condition. Holotype with abdomen glued on card under specimen, and genitalia glued on transparent card.

\section{Ptesimopsia gracilis Rosa, 2004: 227}

Holotype: 1 đิ. Canindé do S. Fco (SE), (Faz. Jaburú), 17.VIII. 2000 am3. Ianuzzi, L. col. (Malaise); [on red] Holótipo; Proj. Xingó Biodiversidade, Área 3; 311.

Conservation status: Good condition. Genitalia in microvial with glycerin under specimen.

\section{Ptesimopsia luscinia Costa, 1975b: 61}

Holotype: 1 đ . Argentina, Missiones, Iguasú XII.1957, A. Martinez leg.; [on red] Ptesimopsia luscinia sp. nov., holótipo, C. Costa det. 1975.

Conservation status: Good condition; genitalia and last abdominal segments glued on transparent card under specimen.

Paratype: 1 ठิ. Porto Alegre, 1962; [on red] Ptesimopsia luscinia sp. nov., parátipo, C. Costa det. 1975.

Conservation status: Good condition; both antennae broken.

Paratype: 1[unknown sex]. S. Salvador R. S., 1960[on red] Ptesimopsia luscinia sp. nov., parátipo, C. Costa det. 1975.

Conservation status: Good condition.

Paratype: 1[unknown sex]. URUGUAY- Canelones, La Floresta, 15.I.1958, M. A. Monné; M.; [on red] Ptesimopsia luscinia sp. nov., parátipo, C. Costa det. 1975.

Conservation status: Good condition.

Paratype: 1[unknown sex]. São Leopoldo; J. W. Stahl; Riksmuseum Stockolm; [on red] Ptesimopsia luscinia sp. nov., parátipo, C. Costa det. 1975.

Conservation status: Good condition.

Paratype: 1[unknown sex]. Rio Grande do Sul, Pe. Buck leg.; MA; [on red] Ptesimopsia luscinia sp. nov., parátipo, C. Costa det. 1975.

Conservation status: Good condition.

Paratype: 1 [unknown sex]. Porto Alegre, RS, XII-1953, Buck leg.; IB; [on red] Ptesimopsia luscinia sp. nov., parátipo, C. Costa det. 1975.

Conservation status: Good condition. 
Paratype: 1[unknown sex]. S. Brasilien, Porto Alegre; 10219; Staatl. Museum fur Tierkunde. Dresden; [on red] Ptesimopsia luscinia sp. nov., parátipo, C. Costa det. 1975.

Conservation status: Good condition.

Paratype: 1 + . 1921, Rio Grande do Sul, Porto Alegre; [on red] Ptesimopsia luscinia sp. nov., parátipo, C. Costa det. 1975.

Conservation status: Good condition. Genitalia and last abdominal segments glued on transparent card under specimen.

Paratype: 1[unknown sex]. Tacuarembé, $\mathrm{A}^{\circ}$ Laureles, 13.15. XII. [1]965, Monné col.; M; [on red] Ptesimopsia luscinia sp. nov., parátipo, C. Costa det. 1975.

Conservation status: Good condition.

Paratype: 1[unknown sex]. URUGUAY- Artigas, Rio Cuareim, Picada del Negro Muerto, Sepulturas, 16-XII-1957, C. S. Carbonell; M; [on red] Ptesimopsia luscinia sp. nov., parátipo, C. Costa det. 1975.

Conservation status: Good condition.

Paratypes: 2 [unknown sex]. Arroyo Los Tapes, Lavallega—Uruguay, 5.1. 1949, P. R. San Martin; M; [on red] Ptesimopsia luscinia sp. nov., parátipo, C. Costa det. 1975.

Conservation status: Good condition.

Paratypes: 3[unknown sex]. Rio Uruguay, Salto Grande Salto, 18.XII.1956-Uruguay, F. Azambuya; M; [on red] Ptesimopsia luscinia sp. nov., parátipo, C. Costa det. 1975.

Conservation status: Good condition. One with last abdominal segments glued on transparent card under specimen.

Paratypes: 2 [unknown sex]. Idem, 25.XII.1956.

Conservation status: Good condition.

Paratype: 1 [unknown sex]. Queb. De Los Cuervos 33, S. Taguato; IB; 683;[on red] Ptesimopsia luscinia sp. nov., parátipo, C. Costa det. 1975.

Conservation status: Good condition.

Paratypes: 5 [unknown sex]. URUGUAY-Soriano, Arroyo Cololó, 20.I.1964, C. S. Norey; M; [on red] Ptesimopsia luscinia sp. nov., parátipo, C. Costa det. 1975.

Conservation status: Good condition. One specimen with abdomen glued on a card under specimen and genitalia into microvial with glycerin, and another specimen with last abdominal segments glued on transparent card under specimen.

Paratype: 1 [unknown sex]. Colonia Gral Artigas, Artigas-Uruguay, XII.1956, F. Azambuya; M;[on red] Ptesimopsia luscinia sp. nov., parátipo, C. Costa det. 1975.

Conservation status: Good condition.

Paratype: 1 [unknown sex]. 1053; Paraguay, Hohenau. Alto Paraná, H. Jacob. B.M. 1954-814; BM; [on red] Ptesimopsia luscinia sp. nov., parátipo, C. Costa det. 1975.

Conservation status: Good condition.

Paratype: 1 [unknown sex]. Argentina, Chaco, Roque, Saenz Peña. 1932, K J Hayward, B.M. 1933-58; BM; [on red] Ptesimopsia luscinia sp. nov., parátipo, C. Costa det. 1975.

Conservation status: Good condition.

Paratype: 1 [unknown sex]. Asuncion-Central, Paraguay, IX.1956, F. Isias-Col.; M; [on red] Ptesimopsia luscinia sp. nov., parátipo, C. Costa det. 1975.

Conservation status: Good condition. Last abdominal segments glued on transparent card under specimen.

Paratype: 1 [unknown sex]. Estancia la Noria, Rio San Javier, Santa Fe, Argentine, G. E. Bryant, 12.xii.1911; BM;

G. Bryant Coll. 1919-147; [on red] Ptesimopsia luscinia sp. nov., parátipo, C. Costa det. 1975.

Conservation status: Good condition.

\section{Genus Pyrearinus Costa, 1975}

Pyrearinus adustus Costa, 1978: 193

Holotype: 1 ठ઼. Bahia, Braz. X.-1923; Bom Jesus dos Minas; Mus. Exped., O. Farrington Col.; CNHM; [on red] Pyrearinus adustus sp. n. holótipo, C. Costa det. 1978.

Conservation status: Good condition; abdomen and genitalia glued on card under specimen. 
Paratype: 1 ठ. Idem.

Conservation status: Idem.

Pyrearinus baliolus Costa, 1978: 194

Holotype: 1 đ. Blanquillo, I.1947, A. Mesa col.; [red] Holótipo; [on red] Pyrearinus baliolus sp. n. holótipo, C. Costa det. 1978; IB.

Conservation status: Good condition; abdomen and genitalia glued on card under specimen.

Paratype: $1 \delta$. S. de la Aurora v. Platón-Riviera, Uruguay-22-25.II.1966, F. Acharai col.; M; [red] Holótipo; [on red] Pyrearinus baliolus sp. n. holótipo, C. Costa det. 1978.

Conservation status: Good condition; abdomen and genitalia glued on card under specimen.

Pyrearinus brunneus Costa, 1978: 196

Holotype: 1 o. Argentina, Stgo. del Estero, El Pinto, XI-1956; CDA; [on red] Holótipo; [on red] Pyrearinus brunneus sp. n. holótipo, C. Costa det. 1978.

Conservation status: Good condition; genitalia glued on transparent card under specimen.

Paratypes: 3 [unknown sex]. Republ. Argentine, Chaco de Santiago del Entero. Rio Dulce; MNHNP; [on red] Pyrearinus brunneus sp. n. paratipo, C. Costa det. 1978.

Conservation status: Good condition.

Paratypes: 2 [unknown sex]. Republ. Argentine, Rio Parana, Territoire des Missiones; MNHNP; [on red] Pyrearinus brunneus sp. n. parátipo, C. Costa det. 1978.

Conservation status: Good condition.

Paratypes: 2 [unknown sex]. Uruguay-Artigas, Ao Catalnau Chico, 23.I.1958; C. Puques; M; [on red] Pyrearinus brunneus sp. n. parátipo, C. Costa det. 1978.

Conservation status: Good condition.

Paratypes: 1우, 3 [unknown sex]. Argentina, Stgodel Estero, El Pinto, XI. 1956; CDA; [on red] Pyrearinus brunneus sp. n. parátipo, C. Costa det. 1978.

Conservation status: Good condition; female genitalia glued on card under specimen.

Paratype: 1 [unknown sex]. Argentina, Coll. Jensen- Baarup; Zool. Mus. Kobenhavn; [on red] Pyrearinus brunneus sp. n. parátipo, C. Costa det. 1978; UZM.

Conservation status: Good condition.

Paratype: 1 [unknown sex]. Mus. Hauschild, 15- [illegible]- 1914; Rio Gde do Sul; UZM; [on red] Pyrearinus brunneus sp. n. parátipo, C. Costa det. 1978.

Conservation status: Good condition.

Paratype: 1 [unknown sex]. Vichadero-Rivera, Uruguay 19.11.961, Carbonell \& Colzolessi; [on red] Pyrearinus brunneus sp. n. parátipo, C. Costa det. 1978.

Conservation status: Good condition.

Pyrearinus castaneus Costa, 1978: 199

Holotype: 1 đ̊. Linhares, ES-Brasil, X.1972, P. C. Elias; [on red] Holótipo; [on red] Pyrearinus castaneus sp. n., holótipo, C. Costa det. 1978.

Conservation status: Good condition; abdomen and genitalia glued on cards under specimen (genitalia on transparent card).

Paratypes: 9 [unknown sex]. Linhares, ES—Brasil, X.1972, P. C. Elias [on red] Pyrearinus castaneus sp. n., parátipo, C. Costa det. 1978.

Conservation status: Good condition; one specimen with abdomen and genitalia glued on cards under specimen (genitalia on a transparent card), and one with genitalia into microvial with glycerin.

Paratype: 1 [unknown sex]. Ribeirão Preto, SP-Brasil, VII.1955, M.P. Barreto col.; [on red] Pyrearinus castaneus sp. n., parátipo, C. Costa det. 1978.

Conservation status: Good condition.

Paratype: 1 [unknown sex]. Anno 1907, São Paulo, E. R. [illegible] da Serra; [on red] Pyrearinus castaneus sp. n., parátipo, C. Costa det. 1978.

Conservation status: Good condition.

Paratype: 1 [unknown sex]. Bahia; Pyrophorus [illegible] Germ., [illegible], Fleutiaux vld.; Riksmuseum Stockolm; [on red] Pyrearinus castaneus sp. n., parátipo, C. Costa det. 1978. 
Conservation status: Good condition.

Paratype: 1 [unknown sex]. E. Santo, Guarajari, X.[1]942; Pyrophorus phosphoreus Germ., A. Cobos det. 1958; [on red] Pyrearinus castaneus sp. n., parátipo, C. Costa det. 1978.

Conservation status: Good condition.

Paratype: 1 [unknown sex]. Rio grand do Sul; CNHM 1955, Karl Brancsik Colln. ex Eduard Knirsch; CNHM; [on red] Pyrearinus castaneus sp. n., parátipo, C. Costa det. 1978.

Conservation status: Good condition.

Paratype: 1 [unknown sex]. Bochiol; Collection E. Candéze; Pyrophorus lampadion Ill., dét. F. Cándeze; [on red] Pyrearinus castaneus sp. n., parátipo, C. Costa det. 1978; Coll. MZSP, São Paulo BR, Ex coll. ISNB.

Conservation status: Good condition.

Paratype: $1 \delta$. Vila Victoria, Prov. de Bahia, Ch. Pujol 1890; [on red] Pyrearinus castaneus sp. n., parátipo, C. Costa det. 1978; MNHNP.

Conservation status: Good condition; genitalia and abdomen glued on card under specimen.

Paratype: 1 đ . Cachimbó, Prov. de Bahia, Ch. Pujol 1890; [on red] Pyrearinus castaneus sp. n., parátipo, C. Costa det. 1978; MNHNP.

Conservation status: Good condition; genitalia and abdomen glued on card under specimen.

Paratypes: 1 ठ, 1 q. Minas Geraes, Fruhstorfer; Museum Paris, 1952, Coll. R. Oberthur; MNHNP; [on red] Pyrearinus castaneus sp. n., parátipo, C. Costa det. 1978; MNHNP.

Conservation status: Good condition; genitalia glued on transparent card under specimen.

Paratype: 1 ô. Brasilia, Mus. Lange; Seckenberg Museum, Frankfurt/Main; [on red] Pyrearinus castaneus sp. n., parátipo, C. Costa det. 1978; MNHNP.

Conservation status: Good condition; genitalia and abdomen glued on card under specimen.

Paratype: 1 ठ․ IB; [on red] Pyrearinus castaneus sp. n., parátipo, C. Costa det. 1978; MNHNP.

Conservation status: Good condition; genitalia and abdomen glued on transparent card under specimen.

Pyrearinus cereus Costa, 1978: 200

Paratype: 1 đ . Bahia; Reed; [on red] Pyrearinus vescus parátipo, C. Costa det. 1978.

Conservation status: Bad condition; abdomen and head glued on card and genitália glued on another transparent card.

Paratype: 1 [unknown sex]. Reed, Bahia; Coll. Janson; BM; [on red] Pyrearinus vescus parátipo, C. Costa det. 1978.

Conservation status: Good condition.

Paratype: $1 \delta$. Uruçuca-BA, 13.12.47, Col. P. Silva; IB; [on red] Pyrearinus vescus parátipo, C. Costa det. 1978. Conservation status: Good condition; abdomen glued on card and genitália glued on transparent card.

\section{Pyrearinus cinnameus Costa, 1978: 201}

Holotype: 1 ठ ${ }^{7}$. Camapuã, MT X.67; [on red] Pyrearinus cinnameus sp. n. holótipo, C. Costa det. 1978; 5676.

Conservation status: Good condition; abdomen and genitalia glued on card under specimen.

Paratype: 1 ㅇ, $1 \delta^{\lambda}, 1$ [unknown sex]. Idem, parátipo.

Conservation status: Good condition; abdomen and genitalia of one male and one female glued on card under specimen.

Paratype: 1 [unknown sex]. Rio Grande do Sul; 5663; Porto Alegre, 1965; [on red] Pyrearinus cinnameus sp. n. holótipo, C. Costa det. 1978.

Conservation status: Good condition.

Paratype: 1 [unknown sex]. Rio Grande do Sul; 5663; Porto Alegre, 1965; [on red] Pyrearinus cinnameus sp. n. holótipo, C. Costa det. 1978.

Conservation status: Good condition.

Paratype: 1 [unknown sex]. Rio Grande do Sul, 5660; [on red] Pyrearinus cinnameus sp. n. holótipo, C. Costa det. 1978.

Conservation status: Good condition; abdomen glued on card under specimen. 
Paratypes: 5 [unknown sex]. Rio Gr. do Sul; Riksmuseum Stockolm; [on red] Pyrearinus cinnameus sp. n. holótipo, C. Costa det. 1978.

Conservation status: Good condition; abdomen of one specimen glued on card under it.

Paratype: 1 [unknown sex]. Rio Gr. de Sul; Walme; Pyrophorus, Fleutiaux det.; [on red] Pyrearinus cinnameus sp. n. holótipo, C. Costa det. 1978.

Conservation status: Good condition.

Pyrearinus coctilis Costa, 1978: 202

Holotype: 1 đ. Faz. Nova Orlandia, Jataí, Go—Brasil, I.1964—Martins, Morgante \& Silva; [on red] Pyrearinus coctilis holótipo, C. Costa det. 1978.

Conservation status: Good condition; abdomen glued on card and genitália glued on transparent card.

Paratypes: 3 [unknown sex]. Faz. Nova Orlandia, Jataí, Go—Brasil, I.1964-Martins, Morgante \& Silva; [on red] Pyrearinus coctilis parátipo, C. Costa det. 1978.

Conservation status: Good condition; one with abdomen glued on card.

Paratype: 1 [unknown sex]. 22-XI-29, Matto Grosso, Murtinho, Spitz leg.; [on red] Pyrearinus coctilis parátipo, C. Costa det. 1978.

Conservation status: Good condition.

Paratype: 1 [unknown sex]. Diamantino, MT-Brasil, 14-XI.1966, Lenko e Pereira; [on red] Pyrearinus coctilis parátipo, C. Costa det. 1978.

Conservation status: Good condition.

Paratypes: 1 Ô, 1 [unknown sex]. Porto Velho, Rio Tapirapé, MT, 30.XII.1964, R.T. Lima col.; [on red] Pyrearinus coctilis parátipo, C. Costa det. 1978.

Conservation status: Good condition; male with abdomen and genitalia glued on transparent card.

Pyrearinus flatus Costa, 1978: 203

Holotype: 1 ठ . Castilho, mar. esq., r. Paraná, SP. X.1964, Exp. Depto. Zool.; [on red] holótipo; [on red] Pyrearinus flatus sp. nov. C. Costa det. 1978, holótipo.

Conservation status: Good condition.

Paratypes: 7. Brasil; Coll. Schwarz; Diech Entomol. Institut Berlin; DEIE; [on red] Pyrearinus flatus sp. nov. C. Costa det. 1978, parátipo.

Conservation status: Good condition.

Paratypes: 2 [unknown sex]. XI. 1944, São Paulo, N. Horizonte, col. Araujo; IB; [on red] Pyrearinus flatus sp. nov. C. Costa det. 1978, parátipo.

Conservation status: Good condition. Abdomen glued on card under one specimen.

Paratype: 1 ô. San Bernardino, A. Fischer, S. G., 31. I. 1913; Seckenberg Museum, Frankfurt/ Main; [on red] Pyrearinus flatus sp. nov. C. Costa det. 1978, parátipo; SMF.

Conservation status: Good condition; abdomen glued on card under specimen; genitalia glued on transparent card under specimen.

Paratype: 1 [unknown sex]. Paraguay; MNHNP; [on red] Pyrearinus flatus sp. nov. C. Costa det. 1978, parátipo.

Conservation status: Good condition.

Paratype: 1 [unknown sex]. Três Lagoas, MT, mar. Esq. Rio Sucuriu, Faz. Canaã, 26.X-4-XI, F. Lane col. 1970; [on red] Pyrearinus flatus sp. nov. C. Costa det. 1978, parátipo.

Conservation status: Good condition.

Paratype: 1 [unknown sex]. Chapada, Brazil, Acc. No, 2966; Nov.; CM; [on red] Pyrearinus flatus sp. nov. C. Costa det. 1978, parátipo.

Conservation status: Good condition; abdomen glued on card under specimen; genitalia glued on transparent card under specimen. 
Pyrearinus fragilis Costa, 1978: 206

Holotype: 1 đ̊. Mangabeira, Mocajuba, Pará, Brasil, XII.1952, Orlando Rêgo; [on red] holótipo; [on red] Pyrearinus fragilis sp. nov. C. Costa det. 1978 holótipo; CS; B5.

Conservation status: Good condition; abdomen glued on card under specimen; genitalia glued on transparent card under specimen.

Paratype: 1 ô. Coleção Campos Seabra; Mangabeira, Mocajuba, Pará, Brasil, , I-1953, Orlando Rego; CS; [on red] Pyrearinus fragilis sp. nov. C. Costa det. 1978 parátipo.

Conservation status: Good condition.

Paratypes: 2 [unknown sex]. Coleção Campos Seabra; Mangabeira, Mocajuba, Pará, Brasil, XII-1952, Orlando Rego; CS; [on red] Pyrearinus fragilis sp. nov. C. Costa det. 1978 parátipo.

Conservation status: Good condition.

Paratype: 1 [unknown sex]. CXXX; CS; [on red] Pyrearinus fragilis sp. nov. C. Costa det. 1978 parátipo.

Conservation status: Good condition.

Pyrearinus fulvescens Costa, 1978: 205

Paratype: 1 $\widehat{~}$. Brésil, Mafra; El 63, M., 2; Museum Paris, 1952, Coll. R, Oberthur; MNHNP; [on red] Pyrearinus fulvescens sp. nov. parátipo, C. Costa det. 1978.

Conservation status: Good condition; abdomen and genitalia glued on transparent card.

Pyrearinus fulvus Costa, 1978: 206

Holotype: 1 đ̂. Faz. Pau D’Alho, Itú-São Paulo, Brasil- I- 953, Col: U. Martins; [on red] Pyrearinus fulvus sp. nov. holótipo, C. Costa det. 1978; [on red] Holótipo; U2.

Conservation status: Good condition; abdomen and genitalia glued on transparent card.

Pyrearinus latus Costa, 1978: 210

Paratypes: 1우 1 $\delta^{\lambda}, 2$ [unknown sex]. Chapada, Brazil, Ace. No. 2966; nov; CM; [on red] Pyrearinus latus sp. n. parátipo, C, Costa det. 1978.

Conservation status: Good condition; male and female with genitalia glued on transparent card under specimen.

Pyrearinus luscinus Costa, 1978: 213

Holotype: $1 \hat{\jmath}$. Instituto Oswaldo Cruz Zona da N. O. B., Salobra, 18-29,x, 38; [on red] Holótipo; [on red] Pyrearinus luscinus sp. n. holótipo, C. Costa det, 1978.

Conservation status: Good condition; abdomen glued on card under specimen; genitália glued on transparent card under specimen.

Paratypes: 4 [unknown sex]. Instituto Oswaldo Cruz Zona da N. O. B., Salobra, 18-29,x,38; [on red] Pyrearinus luscinus sp. n. parátipo, C. Costa det, 1978.

Paratypes: 1 đ̃, 2 [unknown sex]. Coleção M. P. Barreto, 1969; Ribeirão Preto (Tamanduá), X.1954, Barreto col.; [on red] Pyrearinus luscinus sp. n. parátipo, C. Costa det, 1978.

Conservation status: Good condition; one male with abdomen glued on card under specimen, and genitalia glued on transparent card under specimen.

\section{Pyrearinus micatus Costa, 1978: 214}

Holotype: 1 $\widehat{~}$. Mairiporã, SP, Brasil, 4-13. I. 1967, C. Costa col.; [on red] Holótipo; [on red] Pyrearinus micatus sp. n. holótipo, C. Costa det. 1978.

Conservation status: Good condition.

Paratypes: 15 [unknown sex]. Mairiporã, SP, Brasil, 4-13. I. 1967, C. Costa col.; [on red] Pyrearinus micatus sp. n. parátipo, C. Costa det. 1978. 
Conservation status: Good condition.

Paratypes: 1 $\delta^{\lambda}, 4$ [unknown sex]. Três Lagoas, MT, marg. esq. Rio Sucuriu, Faz. Canaã, I. 1967, F. Lane col.; [on red] Pyrearinus micatus sp. n. parátipo, C. Costa det. 1978.

Conservation status: Good condition; one male with abdomen glued on card under specimen, and genitalia on transparent card.

Paratypes: 8 [unknown sex]. São Paulo, Ypiranga; [on red] Pyrearinus micatus sp. n. parátipo, C. Costa det. 1978.

Conservation status: Good condition. One specimen with prothorax detached of the rest of the body.

Paratype: 1 [unknown sex]. São Paulo, Capital, col. A.C.M., XII. 33; [on red] Pyrearinus micatus parátipo, C. Costa det. 1978.

Conservation status: Good condition.

Paratype: 1 [unknown sex]. 3.V.32, São Paulo, Alto da Lapa, Col. B.L.R.; [on red] Pyrearinus micatus parátipo, C. Costa det. 1978.

Conservation status: Good condition.

Paratypes: 4 [unknown sex]. Faz. Pau D'Alho, Itu—SP-Brasil, 1-8.1970, U. Martins col.; [on red] Pyrearinus micatus parátipo, C. Costa det. 1978.

Conservation status: Good condition.

Paratypes: 6 [unknown sex]. Faz. Pau D’Alho, Itu, São Paulo, Brasil, XII—1970, U. Martins col.; [on red] Pyrearinus micatus parátipo, C. Costa det. 1978.

Conservation status: Good condition.

Paratypes: 18 [unknown sex]. Luiz Antonio_SP, (Faz. Jataí), 18.I.1972, E.X. Rabello col.; [on red] Pyrearinus micatus parátipo, C. Costa det. 1978.

Conservation status: Good condition.

Paratype: 1 [unknown sex]. J. P. Guarulhos, IV-1944, R. Morano col., F. Pereira o F.; [on red] Pyrearinus micatus parátipo, C. Costa det. 1978.

Conservation status: Good condition.

Paratypes: 7 [unknown sex]. Faz. Morongaba, Cabreúva, S. Paulo, 2.2.1966, C. Costa; [on red] Pyrearinus micatus parátipo, C. Costa det. 1978.

Conservation status: Good condition.

Paratypes: 3 [unknown sex]. Barueri, São Paulo, Brasil, XII.1965, K. Lenko col.; [on red] Pyrearinus micatus parátipo, C. Costa det. 1978.

Conservation status: Good condition.

Paratypes: 7 [unknown sex]. Faz. Pau D’Alho, Itú, S. Paulo, Brasil, 27. XII.[1]957, Col: U. Martins; [on red] Pyrearinus micatus parátipo, C. Costa det. 1978.

Conservation status: Good condition.

Paratypes: 2 [unknown sex]. Idem, 7.XII.1960.

Conservation status: Idem.

Paratypes: 1 [unknown sex]. Idem, 1.XII.1960.

Conservation status: Idem.

Paratypes: 11 [unknown sex]. Campinas, SP (Souzas), J. Guérin of; [on red] Pyrearinus micatus parátipo, C. Costa det. 1978 [ 5 collected on II-1946, and 6 collected on XII-1946].

Conservation status: Good condition.

Paratypes: 8 [unknown sex]. Goiatuba, Go, III-1946, J. Guérin of; IB; ?; [on red] Pyrearinus micatus parátipo,

C. Costa det. 1978.

Conservation status: Good condition.

Paratype: 1 [unknown sex]. Goiatuba, GO, III—1976, J. Guérin o F; cinerarius ou candens?; [on red] Pyrearinus micatus parátipo, C. Costa det. 1978.

Conservation status: Good condition.

Paratype: 1 [unknown sex]. Pyrophorus, Fleutiaux det.; [on red] Pyrearinus micatus parátipo, C. Costa det. 1978. Conservation status: Good condition.

Paratypes: 5 [unknown sex]. Coleção J. Lane; Sto. Amaro, S. Paulo, J. Lane col.; [on red] Pyrearinus micatus parátipo, C. Costa det. 1978.

Conservation status: Good condition. 
Paratypes: 3 [unknown sex]. I- 923, São Paulo, Itatiba; [on red] Pyrearinus micatus parátipo, C. Costa det. 1978. Conservation status: Good condition.

Paratypes: 8 [unknown sex]. Faz. Pau D’Alho, Itu—SP—Brasil, 1-8.I.1970, U. Martins col.; [on red] Pyrearinus micatus parátipo, C. Costa det. 1978.

Conservation status: Good condition.

Paratype: 1 [unknown sex]. 2028; [on red] Pyrearinus micatus parátipo, C. Costa det. 1978.

Conservation status: Good condition.

Paratype: 1 [unknown sex]. Jundiahy, E. S. Paulo; Pyrophorus parallelus Germ, Fleutiaux det.; [on red] Pyrearinus micatus parátipo, C. Costa det. 1978.

Conservation status: Good condition.

Paratype: 1 đ. 13.I.1956, Barueri, S. Paulo, 8280; K. Lenko leg.; Coleção Campos Seabra.; [on red] Pyrearinus micatus parátipo, C. Costa det. 1978.

Conservation status: Good condition; abdomen and genitalia glued on transparent card under specimen.

Paratype: 1 o. 455; Pyrophorus phosphoreus Linn.; S.F.; [on red] Pyrearinus micatus parátipo, C. Costa det. 1978.

Conservation status: Good condition; abdomen and genitalia glued on transparent card under specimen.

Paratypes: 7 [unknow]. Souza-Campinas, Est. de S. Paulo, I-1946, J. Guér. Of, Col. I. Biológico; [on red] Pyrearinus micatus parátipo, C. Costa det. 1978.

Conservation status: Good condition. One with prothorax separated from the rest of the body.

Paratype: 1 [unknown sex]. Piracicaba, SP, XII-1950, Marican leg.; IB; [on red] Pyrearinus micatus parátipo, C. Costa det. 1978.

Conservation status: Good condition.

Paratype: 1 [unknown sex].: Piracicaba, SP, Ex. Col. Ent. E. S. Agr. L. Queiroz, Col. I. Biológico; [on red] Pyrearinus micatus parátipo, C. Costa det. 1978.

Conservation status: Good condition.

Paratypes: 2 [unknown sex]. Ponta Grossa, Paraná, 1944, IB; Coll. J. Guérin, S. Paulo Brasil, 16922 [on red] Pyrearinus micatus parátipo, C. Costa det. 1978.

Conservation status: Good condition.

Paratypes: 20 [unknown sex]. Campinas, J. Guérin col.; IBSP; [on red] Pyrearinus micatus parátipo, C. Costa det. 1978 [8 collected on I.1946, 12 on XII.1946].

Conservation status: Good condition.

Paratype: 1 [unknown sex]. 31.XII.34, Ypiranga, R. Sp.; [on red] Pyrearinus micatus parátipo, C. Costa det. 1978. Conservation status: Good condition.

Paratype: 1 đ̊. E. de S. Paulo, 6992; [on red] Pyrearinus micatus parátipo, C. Costa det. 1978.

Conservation status: Good condition; genitalia glued on transparent card under specimen.

Paratype: 1 ठ․ São Paulo, (Ypiranga) S.P., Brasil, 10.XI.1965, C. Costa col.; [on red] Pyrearinus micatus parátipo,

C. Costa det. 1978.

Conservation status: Good condition; genitalia glued on transparent card under specimen.

Pyrearinus pusillus Costa, 1978: 218

Holotype: 1 $\delta$. Filadelfia, Fer. Col. Chaco, Parag. I.21.56; J. L. Nickel Collector. [on red] Holótipo; [on red] Pyrearinus pussilus holótipo, C. Costa det. 1978; đ̊; CIS.

Conservation status: Good condition; abdomen and genitalia glued on transparent card under specimen.

Paratype: $1 \delta$. Idem, parátipo; light.

Conservation status: Idem.

Pyrearinus ruscus Costa, 1978: 219

Holotype: 1 ठ . S. Paulo, S. Amaro, 5-I-24; [on red] Pyrearinus ruscus holótipo, C. Costa det. 1978; A2.

Conservation status: Good condition; abdomen and genitalia glued on transparent card under specimen. 
Paratype: 1 o. Curytiba, Brasil, Szabo; A. Fenyes Collection; s. f.; Pyrophorus nyctolampis Germ.; [on red] Pyrearinus ruscus parátipo, C. Costa det. 1978.

Conservation status: Idem, only right pro-and mesolegs presents.

Pyrearinus termitilluminans Costa, 1982: 23

Holotype: 1 ठ․ No. 228; [on red] Pyrearinus termitilluminans holotype, C. Costa det. 1982; Brasil—GO, Parque Nacional das Emas, 2-4.X.1981 on Cornitermis mound.

Conservation status: Good condition.

Paratypes: 11 [unknown sex]. Idem, K. H. Redford col. (only 2 specimens with collector's information). Six labelled with 228 and two labelled with 238 .

Conservation status: Good condition; 2 specimens dissected, one with genitalia glued on transparent card under specimen, and another with genitalia into microvial with glycerin. One pupa and 2 larvae at CIC (Coleoptera Immature Collection) conserved on Alcohool $70^{\circ} \mathrm{GL}$.

Pyrearinus vesculus Costa, 1978: 221

Paratype: 1 ㅇ. Ega; BM; [on red] Pyrearinus vesculus parátipo, C. Costa det. 1978.

Conservation status: Good condition; abdomen glued on card under specimen, and genitalia and last abdominal segments glued on another transparent card.

Paratype: 1 ठ․ Ega, Amazonas, Bates; Coll. Janson.; [on red] Pyrearinus vesculus parátipo, C. Costa det. 1978.

Conservation status: Good condition; abdomen glued on card under specimen, and genitalia glued on another transparent card.

Paratype: 1 [unknown sex]. Chiguinda, Ecuador, Buckley; BM; [on red] Pyrearinus vesculus parátipo, C. Costa det. 1978.

Conservation status: Good condition.

Paratype: 1 [unknown sex]. Amazones, Teffé, Dr. Hahnel; Museum Paris, 1952, Coll R. Oberthür; MNHNP; [on red] Pyrearinus vesculus parátipo, C. Costa det. 1978.

Conservation status: Good condition; left foreleg missing; abdomen glued on card under specimen.

Pyrearinus vescus Costa, 1978: 221

Holotype: 1 đ̂. Faz. Cachoeirinha, Jataí, Goiás-Brasil, X.1962, Exp. Dep. Zool.; [on red] Holótipo; [on red] Pyrearinus vescus holótipo, C. Costa det. 1978.

Conservation status: Good condition; abdomen glued on card under specimen and genitália glued on transparent card.

\section{Genus Pyrophorus Billberg, 1820}

Pyrophorus angustus hayekae Costa, 1968: 82

Paratypes: 2 [unknown sex]. Canindé: Suriname: Kayseberg, Zuid R. Surinam Zool. Exped., 1960-62. H. Beatty Leg.; CNHM; [on red] Pyrophorus angustus hayekae subps. n., Parátipo, C. Costa det. 1968.

Conservation status: Good condition.

Paratype: 1 [unknown sex]. Canindé Kaieter BG, 13.VIII.1911; AMNH; [on red] Pyrophorus angustus hayekae subps. n., Parátipo, C. Costa det. 1968.

Conservation status: Idem.

Paratype: 1 [unknown sex]. Upper Mazaruni R., Br Guiana, IX-X.1938; Coll. A.S. Pinkus; AMNH; [on red] Pyrophorus angustus hayekae subps. n., Parátipo, C. Costa det. 1968.

Conservation status: Idem.

Paratype: 1 [unknown sex]. Brasil, A P, J \& Bechyné; Serra Lombard, Limão, 30.8.1961; M.G.; [on red] Pyrophorus angustus hayekae subps. n., Parátipo, C. Costa det. 1968. 
Conservation status: Good condition; genitalia glued on transparent card; left antenna missing. Paratype: 1 [unknown sex]. see notes; British Guiana, Essequibo R., Moraballi Creek. 28-29.IX.1929, Oxf. Univ. Expedn., B. M. 1929-485. 2195; BM; [on red] Pyrophorus angustus hayekae subps. n., Parátipo, C. Costa det. 1968.

Conservation status: Good condition.

Paratype: 1 [unknown sex]. Santarém, Brazil; [on red] Pyrophorus angustus hayekae subps. n., Parátipo, C. Costa det. 1968; CM.

Conservation status: Idem.

Paratype: 1 ठ. Amazonas, Borba (Lago Acara), IX-X.1943 A. Parlo; 171; MN; [on red] Pyrophorus angustus hayekae subps. n., Parátipo, C. Costa det. 1968.

Conservation status: Good condition; genitalia glued on transparent card under specimen.

Paratype: 1 [unknown sex]. Santarem-Pará, II.1921, C, Boy; Km ec; [on red] Pyrophorus angustus hayekae subps. n., Parátipo, C. Costa det. 1968.

Conservation status: Good condition.

Paratype: 1 ठో. Óbidos, 7-8-1912, Ducke; Óbidos, 7.8.1912, Ducke; M. G.; 173; [on red] Pyrophorus angustus hayekae subps. n., Parátipo, C. Costa det. 1968.

Conservation status: Good condition; genitalia glued on transparent card under specimen.

Pyrophorus expeditus Costa, 1972: 225

Paratype: 1 [unknown sex]. Merida, Venezuela; W. Robinson beguest 1929; Property USNM; [on red] Pyrophorus expeditus parátipo C. Costa det. 1970.

Conservation status: Good condition.

Pyrophorus indulcatus Costa, 1972: 208

Paratype: 1 ठ․ W. Indies, 97-175; St. Vincent, W. I., H. H. Smith., 9; nodilucus L. Var; [on red]Paratipo; [on red] Pyrophorus indulcatus parátipo C. Costa det. 1970; BM.

Conservation status: Good condition; abdomen glued on card under specimen.

Paratype: 1 [unknown sex]. St. Vincent, W. I., H. H. Smith., 23; [on red] Paratipo; [on red] Pyrophorus indulcatus parátipo C. Costa det. 1970; BM.

Conservation status: Good condition.

Paratypes: 2 $\delta$. W. Indies, 97-175; St. Vincent, W. I., H. H. Smith., 23; [on red] Paratipo; [on red] Pyrophorus indulcatus parátipo C. Costa det. 1970; BM.

Conservation status: Good condition; genitalia glued on transparent card under specimen.

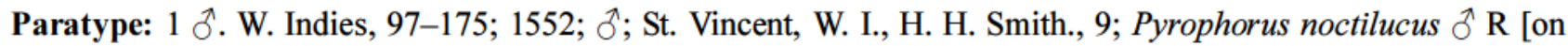
red] Paratipo; [on red] Pyrophorus indulcatus parátipo C. Costa det. 1970; BM.

Conservation status: Bad condition; left elytra and abdomen missing; genitalia glued on card in separated pin. Paratype: 1 [unknown sex]. W. Indies, 97-175; St. Vincent, W. I., H. H. Smith., 2; Pyrophorus noctilucus ठิ R [on red] Paratipo; [on red] Pyrophorus indulcatus parátipo C. Costa det. 1970; BM.

Conservation status: Good condition.

Pyrophorus ingens Costa, 1972: 224

Paratype: 1 đ. Panama; J.J. DuBois Collection; CIS; [on red] Pyrophorus ingens parátipo C. Costa det. 1970.

Conservation status: Good condition; genitalia glued on transparent card under specimen.

Paratype: 1 [unknown sex]. Carilla, V. 911; Museum Paris, Costa Rica, Paul Serre 1920; MNHNP; [on red] Pyrophorus ingens parátipo C. Costa det. 1970.

Conservation status: Idem.

Paratype: 1 [unknown sex]. Guayabillos, SW—Abhang, Irazu 2200m; Property USNM; [on red] Pyrophorus ingens parátipo C. Costa det. 1970.

Conservation status: Idem. 
Paratype: 1 [unknown sex]. S. W. Abhang, Irazu 2200m; T. Assmann Coll; Nevermann Collection 1940; [on red] Pyrophorus ingens parátipo C. Costa det. 1970.

Conservation status: Idem.

Pyrophorus jocundus Costa, 1972: 211

Paratype: 1 đ. Panama; J. J. Dubois Collection; ô; [on red] Paratipo; [on red] Pyrophorus jocundus parátipo C. Costa det. 1970.

Conservation status: Good condition; left antenna glued on triangle under specimen.

Paratype: 1 [unknown sex]. Chiriqui Prov., Panama, XII.10.37; [on red] Paratipo; [on red] Pyrophorus jocundus parátipo C. Costa det. 1970; AMNH.

Conservation status: Good condition; both antennae missing.

Paratypes: 3 ô. Potrerilles, Panama, IV.28-V.8.33; Van Dyke Collection; [on red] Paratipo; [on red] Pyrophorus jocundus parátipo C. Costa det. 1970; S. F.

Conservation status: Good condition; two paratypes with genitalia glued on transparent card under specimen. Paratype: $1 \hat{\delta}$. Poterillos, Panama; DV Brown; Van Dyke Collection; [on red] Paratipo; [on red] Pyrophorus jocundus parátipo C. Costa det. 1970; S. F.

Conservation status: Good condition; genitalia glued on transparent card under specimen.

Paratype: 1 ठ․ Poterillos, Panama, I.1934; D. V. Brown Collector; Pyrophorus gtrabus Germ.; [on red] Paratipo; [on red] Pyrophorus jocundus parátipo C. Costa det. 1970; S. F.

Conservation status: Good condition; genitalia glued on transparent card under specimen.

Paratype: 1 o. Porterillos, Panama, IV.27.54; Pres by RF Starnitaky; [on red] Paratipo; [on red] Pyrophorus jocundus parátipo C. Costa det. 1970; S. F.; CIS.

Conservation status: Good condition; genitalia and part of antenna, each one, glued on transparent card under specimen.

Pyrophorus mellifluus Costa, 1972: 211

Paratype: 1 [unknown sex]. Cuba, La Havane; IRSN; [on red] Paratype; [on red] Pyrophorus mellifluus sp. n., parátipo, C. Costa det. 1970.

Conservation status: Good condition.

Paratype: 1 [unknown sex]. Cuba, Cinza dezanata [ilegible]; ô; L 15 B; IB; [on red] Paratype; [on red] Pyrophorus mellifluus sp. n., parátipo, C. Costa det. 1970.

Conservation status: Good condition.

Paratype: 1 [unknown sex]. Cuba, Halquen; CNHM 1955, Karl Bransik Colln, ex Eduard Knirsch; CNHM; [illegible] Cand., O. Schwarz det. 1901;[on red] Paratype; [on red] Pyrophorus mellifluus sp. n., parátipo, C. Costa det. 1970.

Conservation status: Good condition.

Paratype: $1 \delta$. Baños de Ciego, Montero, Sarita Clara, Cuba, V/10/10; AMNHN ; [on red] Paratype; [on red] Pyrophorus mellifluus sp. n., parátipo, C. Costa det. 1970.

Conservation status: Good condition; genitalia glued on transparent card under specimen.

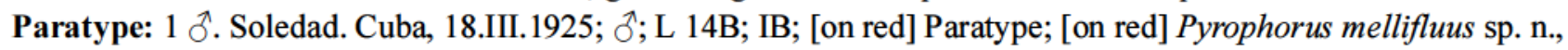
parátipo, C. Costa det. 1970.

Conservation status: Good condition.

Paratype: 1 [unknown sex]. Cuba, Guantanamo, Collection Le Moult; Pyrophorus phosphorescens; IRSN; [on red] Paratype; [on red] Pyrophorus mellifluus sp. n., parátipo, C. Costa det. 1970.

Conservation status: Good condition.

Paratype: 1 [unknown sex]. Nueva Gerona, Isle of Pines. G. Link, Coll; March 17 1973; CM; [on red] Paratype; [on red] Pyrophorus mellifluus sp. n., parátipo, C. Costa det. 1970.

Conservation status: Good condition; genitalia glued on transparent card under specimen.

Paratype: 1 [unknown sex]. Nueva Gerona, Isle of Pines. G. Link, Coll; March 1913; Carn Mus. Acc. 4865; CM; [on red] Paratype; [on red] Pyrophorus mellifluus sp. n., parátipo, C. Costa det. 1970. 
Conservation status: Good condition.

Paratype: 1 [unknown sex]. Hayti, Parish 1899; Sharp Coll., 1905-313; Pyrophorus noctilucus Linn.; BM; [on red] Paratype; [on red] Pyrophorus mellifluus sp. n., parátipo, C. Costa det. 1970.

Conservation status: Good condition.

Paratype: 1 [unknown sex]. Habana, Cuba; MNHNP; [on red] Paratype; [on red] Pyrophorus mellifluus sp. n., parátipo, C. Costa det. 1970.

Conservation status: Good condition.

Paratype: 1 q. Haiti; Coll. Kraatz; Dtsch. Ent. Inst. Eberswaide; DEIE; [on red] Paratype; [on red] Pyrophorus mellifluus sp. n., parátipo, C. Costa det. 1970.

Conservation status: Good condition; genitalia and abdomen glued on transparent card under specimen.

Paratype: 1 ठ․ St. Domingo, 1906, C. Rainkiaer; [on red] Paratype; [on red] Pyrophorus mellifluus sp. n., parátipo, C. Costa det. 1970.

Conservation status: Good condition.

Paratype: $1 \delta$. Cuba. Ex. Musaeo A. Sallé, 1897; MNHNP; [on red] Paratype; [on red] Pyrophorus mellifluus sp. n., parátipo, C. Costa det. 1970.

Conservation status: Good condition.

Paratype: $1 \delta$. Antilles, [illegible]; Fry Coll. 1905-100; BM; [on red] Paratype; [on red] Pyrophorus mellifluus sp. n., parátipo, C. Costa det. 1970.

Conservation status: Good condition; abdomen glued on transparent card under specimen.

Paratype: 1 [unknown sex]. Haiti, Heyne; Coll. Schwarz; Plagiophthalmus Germ.; Plagiophthalmus Germ.; Dtsch. Ent. Inst. Eberswaide; DEIE; [on red] Paratype; [on red] Pyrophorus mellifluus sp. n., parátipo, C. Costa det. 1970.

Conservation status: Good condition.

Paratype: 1 đ̆. Sa. Vicente de Vinales, Cuba [ilegible]; MCZ; [on red] Paratype; [on red] Pyrophorus mellifluus sp. n., parátipo, C. Costa det. 1970.

Conservation status: Good condition.

Paratype: 1 [unknown sex]. Cuba; Ex. Museo A. Sallè, 1897; MNHNP; [on red] Paratype; [on red] Pyrophorus mellifluus sp. n., parátipo, C. Costa det. 1970.

Conservation status: Good condition.

Paratypes: 2 [unknown sex]. Museum Paris, ANTILLES, Sainte Lucie, Bonnecour 1851; MNHNP; [on red] Paratype; [on red] Pyrophorus mellifluus sp. n., parátipo, C. Costa det. 1970.

Conservation status: Good condition.

Paratype: 1 ठ. Puerta Plata, R. Dom. V.20 to VII.5.15; MNHNP; [on red] Paratype; [on red] Pyrophorus mellifluus sp. n., parátipo, C. Costa det. 1970.

Conservation status: Good condition; genitalia glued on transparent card under specimen.

Paratype: 1 [unknown sex]. Habana, Cuba; MNHNP; [on red] Paratype; [on red] Pyrophorus mellifluus sp. n., parátipo, C. Costa det. 1970.

Conservation status: Good condition.

Paratype: 1 [unknown sex]. Cuba, Guantanamo, Roll, Berlin, SW11; [on red] Paratype; [on red] Pyrophorus mellifluus sp. n., parátipo, C. Costa det. 1970.

Conservation status: Good condition.

Paratype: 1 [unknown sex]. Cuba; Guantanamo, O Toherin; Riksmuseum Stockolm; [on red] Paratype; [on red] Pyrophorus mellifluus sp. n., parátipo, C. Costa det. 1970.

Conservation status: Good condition.

Paratype: 1 [unknown sex]. Soledad Sta Clara, Cuba 1932; MCZ; [on red] Pyrophorus mellifluus sp. n., parátipo,

C. Costa det. 1970.

Conservation status: Good condition.

Paratype: $1 \widehat{\delta}$. Cuba [illegible]; $\partial^{\lambda}$; [on red] Pyrophorus mellifluus sp. n., parátipo, C. Costa det. 1970.

Conservation status: Good condition; genitalia glued on transparent card under specimen.

Paratype: 1 [unknown sex]. Cuba; Guantanamo; [on red] Paratype; [on red] Pyrophorus mellifluus sp. n., parátipo,

C. Costa det. 1970.

Conservation status: Good condition. 
Paratype: $1 \hat{\delta}$. Martinique; Pyrophorus plagiophtalmus; P. plagiophtalmus Germ., J. Mouchet det.; MNHNP; [on red] Pyrophorus mellifluus sp. n., parátipo, C. Costa det. 1970.

Conservation status: Good condition; genitalia glued on transparent card under specimen.

Pyrophorus mellitus Costa, 1972: 210

Paratype: 1 đ. Santa Lucia, G. A. Ramage, June. 1889, 97-97; Fonde Sa Sques, April 1889; [on red] Paratype; [on red] Pyrophorus mellitus sp. n. parátipo, C. Costa det. 1970.

Conservation status: Good condition; genitalia glued on transparent card under specimen.

Paratypes: 3 đ. Santa Lucia, G. A. Ramage, June. 1889, 97-67; Fonde Sa Sques, April 1889; [on red] Paratype; [on red] Pyrophorus mellitus sp. n. parátipo, C. Costa det. 1970.

Conservation status: Idem.

Paratype: 1 ठ․ Santa Lucia, G. A. Ramage, June. 1889, 97-67; Fonde Sa Sques, April 1889; đ^; [on red] Paratype; [on red] Pyrophorus mellitus sp. n. parátipo, C. Costa det. 1970.

Conservation status: Good condition.

Paratype: $1 \widehat{\delta}$. Museum Paris, Antilles, Saint Lucie, Bonnecour, 1851; $\widehat{\delta}$; [on red] Paratype; [on red] Pyrophorus mellitus sp. n. parátipo, C. Costa det. 1970.

Conservation status: Good condition.

Paratype: 1 ðో. St. Lucia (Mém. foreset) April, 1956, H. E. Box; Pres, by Com. Inst. Ent. B. M. 1948-146; BM; đ;; [on red] Paratype; [on red] Pyrophorus mellitus sp. n. parátipo, C. Costa det. 1970.

Conservation status: Good condition.

Pyrophorus pisticus Costa, 1972: 221

Paratype: 1 đ. Pacto, 4.500 ft, Ecuador, Dolby-Tyler; 331; G.C. Champion Coll. B.M. 1927-409; [on red] Pyrophorus pisticus paratipo, C. Costa det. 1970; BM.

Conservation status: Good condition; genitalia glued on transparent card under specimen. Abdomen glued on card under specimen.

Paratype: $1 \delta^{\lambda} .2^{\circ}-4^{\circ} \mathrm{S} ; 78^{\circ} \mathrm{W}, \mathrm{X}-\mathrm{XII} .1934,600-950 \mathrm{~m}$; AMNH; [on red] Pyrophorus pisticus paratipo, C. Costa det. 1970.

Conservation status: Idem.

Pyrophorus stupendus Costa, 1972: 216

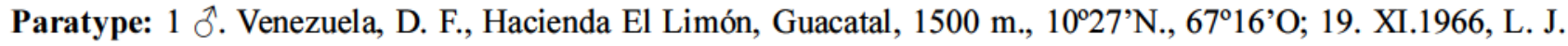
Joly T., J. L. Garcia; [on red] Pyrophorus stupendus parátipo C. Costa det. 1970.

Conservation status: Good condition; genitália glued on transparent card under specimen.

Paratype: 1 đิ. Los Guayabitos. MI.-10-I.1963, Y. Ramirez; MIS; [on red] Pyrophorus stupendus parátipo C. Costa det. 1970.

Conservation status: Good condition; genitalia and abdomen glued on card under specimen.

Paratype: 1 đิ. Borhurata, Ven. 15.III.1940 G. Vivas; Property USNM; [on red] Pyrophorus stupendus parátipo C. Costa det. 1970.

Conservation status: Good condition; genitalia glued on transparent card under specimen.

Pyrophorus validus Costa, 1972: 217

Paratype: 1 [unknown sex]. Equateur, Envd Ambato, R. P. Irenee Blanc; Muséum Paris, 1952, Coll. R. Oberthur; MNHNP; [on red] Pyrophorus validus Costa, 1970, Parátipo.

Conservation status: Good condition; abdomen glued on white card under specimen. Genitalia glued on transparent card under specimen.

Paratype: 1 ठ․ Ex. Musaeo E. Steinheil; Muséum Paris, 1952, Coll. R. Oberthur; MNHNP; [on red] Pyrophorus validus Costa, 1970, Parátipo. 
Conservation status: Idem.

Paratype: $1 \hat{\delta}$. Colombia Caucal, Distrito de Pereira, Roman M Valencia, 1886; MNHNP; [on red] Pyrophorus validus Costa, 1970, Parátipo.

Conservation status: Idem.

Paratype: 1 ภ. Macas, Ecuador, Buckley; Coll. Janson; BM; [on red] Pyrophorus validus Costa, 1970, Parátipo.

Conservation status: Good condition; genitalia glued on transparent card under specimen.

Paratype: $1 \delta$. Zatzayacu, Oriente, Ecuador, VII.30.1934; W. Macintyre, Collection; Ex Coll, L. Lacey; [on red] Pyrophorus validus Costa, 1970, Parátipo; AMNH.

Conservation status: Idem.

Paratype: 1 [unknown sex]. Equateur, Baños, R.P.I. Blanc, 1895; [on red] Pyrophorus validus Costa, 1970, Parátipo; MNHNP.

Conservation status: Good condition.

Pyrophorus veriloquus Costa, 1972: 219

Holotype: $1 \delta$. Guatemala, Conradt; MNHNP; [on red] Pyrophorus veriloquus Holótipo, C. Costa det. 1970; [on red] Holótipo.

Conservation status: Good condition; abdomen glued on white card under specimen; genitalia glued on transparent card under specimen.

Paratypes: 4 ô. Guatemala, Conradt; MNHNP; [on red] Pyrophorus veriloquus parátipo, C. Costa det. 1970.

Conservation status: Good condition. Two specimens with abdomen glued on white card; genitalias glued on transparent cards under specimen.

Paratype: 1 [unknown sex]. Guatemala; Coll. Kraalz; Dtsch. Ent. Inst. Eberwalde; DEIE.

Conservation status: Good condition.

\section{Genus Pyroptesis Costa, 1975}

Pyroptesis gilvus Costa, 1975c: 2

Holotype: 1 đิ. [on red] Holótipo; Brasil, S. Paulo, Salesópolis-Boracéia, 12-14.XI.1969, C. Costa col.; [on red] Pyroptesis gilvus sp. nov., holótipo, C. Costa det. 1974.

Conservation status: Good condition; abdomen glued on card under specimen, genitalia glued on transparent card under specimen.

Additional notes: The larva of the holotype was reared in laboratory. The last larval and pupal instars are deposited at CIC under registration number 10.083.

Paratype: 1 ㅇ. Est. Biol. Boraceia, Salesópolis-S.P., 12.II.1963, L. Silva \& H. Reichardt col.; [on red] Pyroptesis gilvus sp. nov., parátipo, C. Costa det. 1974.

Conservation status: Good condition; genitalia and last abdominal segments glued on transparent card under specimen.

\section{Selatosomus Stephens, 1830}

Selatosomus aeripennis destructor (Brown, 1935a): 129

Paratype: 1 [unknown sex]. Medicine; Hat. Alta, VI.31.1923; F.S. Carr collector; [on yellow] L. aeripennis destructor, No. 3809 Brown.

Conservation status: Good condition.

Additional notes: Described as a subspecies it was elevated to species by Brooks (1960) then returned to subspecies by Lane (1971). Gurjeva (1989) transferred the nominal species to Selatosomus. 
Triplonychus crassifemoris Rosa, 2011: 8

Holotype: 1 đิ. Igarapé Gurupi-Una, Aldeia Araçu, MA, $50 \mathrm{Km}$ E de Canindé, II.1966, Malkin col.; [on red] Holótipo; [on red] Triplonychus crassifemoris Rosa, 2011, Holótipo.

Conservation status: Good condition; specimen glued on white card.

Paratypes: 5 $\hat{\delta}$. Idem.

Conservation status: Good condition. 3 specimens glued on triangles by elytra; one glued on card; one with genitalia dissected in a tube under specimen.

Triplonychus cruspinosus Rosa, 2011: 3

Paratypes: 16 . Brasil, Reserva Ducke, $26 \mathrm{Km}$ NE Manaus, Barbosa, M.G.V., malaise trap [2 collected on II.1995; 10 collected on V.1995; 4 collected on XII.1995]; [on red] Triplonychus cruspinosus Rosa, 2011, Parátipo.

Conservation status: Good condition.

Paratypes: 24 đ. Brasil: Amazonas, Reserva Ducke, 26 km NE Manaus, 28.II.1978, J. Arias \& A. Penny; [on red] Triplonychus cruspinosus Rosa, 2011, Parátipo.

Conservation status: Good condition; glued on white cards and or triangles.

Paratypes: 16 J. Brasil: Amazonas, AM 010, Km 26, Reserva Ducke, Jorge Arias coll.; 12 collected 7.II.1978; 4 collected on 14.II.1978; [on red] Triplonychus cruspinosus Rosa, 2011, Parátipo

Conservation status: Idem.

Triplonychus tibialatus Rosa, 2011: 9

Holotype: 1 ô. 08/XII/1994, BR-MS—Três Lagoas, International Paper, Horto Rio Verde, black light FIT, Eucalyptus grandis stand, Flechtmann, C.A.H. col.; C1945; CRELAT028; [on red] Holótipo; [on red] Triplonychus tibialatus Rosa, 2011, Holótipo.

Conservation status: Good condition; specimen glued on triangle by mesoventrite.

Paratype: 5 . Idem (except by the date of collect: two from 11///1995; two from 08/X/1994; one from 08/XII/ 1994).

Conservation status: Good condition; all glued on triangles by mesoventrite.

\section{Genus Tropihypnus Reitter, 1905}

Tropihypnus rungbongi Stibick, 1968: 172

Paratype: 1 đิ [on a yellow circle] Paratype; Sikkimi, Gopaldhara, Rungbong Vall, H. Stevens; Stevens Coll, B.M. 1916.218.; Paratype, ठ̂, Tropihypnus rungbongi Stibick.

Conservation status: Good condition; glued on white card.

Paratype: 1 ㅇ. Idem, 우.

Conservation status: Idem.

Genus Zorochros Thomson, 1859

Zorochros birganjianus Ohira \& Becker, 1973:70

Paratypes: 1 [unknown sex]. NEPAL nr. Birganj, Lothar $450 \mathrm{ft}, 8 \mathrm{Sept}$ [19]67, Can. Nepal. Exped; [on yellow] Paratype, CNC No. 12766, Zorochrus birganjianus Ohira \& Becker.

Conservation status: Good condition; glued on triangle by right elytra.

Paratypes: 1 [unknown sex . NEPAL nr. Birganj, Lothar $450 \mathrm{ft}, 12 \mathrm{Sept}$. [19]67, Can. Nepal. Exped; [on yellow] Paratype, CNC No. 12766, Zorochrus birganjianus Ohira \& Becker.

Conservation status: Idem. 
Eucnemidae Eschscholtz, 1829

Genus Fornax Laporte, 1835

Subgenus Filifornax Fleutiaux, 1945

Fornax (Filifornax) foersteri Cobos, 1955: 2

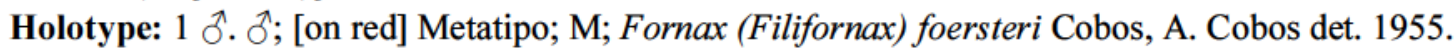
Conservation status: Good condition.

Fornax (Filifornax) proximus Cobos, 1956: 9

Holotype: 1 ठో. [on red] Holótipo; Ponta Grossa, Paraná-Brasil, II.1938, C.A. Camargo col.; Fornax (Filifornax) proximus nov. sp. A. Cobos det. 1955

Conservation status: Good condition.

Genus Pereirella Cobos, 1956

Pereirella paulensis Cobos, 1956: 5

Holotype: 1 đै. [on red] Holótipo; Ypiranga, E.S. Paulo; Pereirella paulensis m[ihi]. nov. sp. A. Cobos det. 1955. Conservation status: Good condition; genitalia glued on card under specimen.

\section{Genus Neodiaeretus Cobos, 1956}

Neodiaeretus brasiliensis Cobos, 1956: 8

Holotype: 1 đ. [on red] Holótipo; São Paulo, Batêa, 2.XII.1940, F. Lane coll.; Neodiaeretus brasiliensis m[ihi]. nov. sp. A. Cobos det. 1955.

Conservation status: Good condition; genitalia glued on card under specimen.

Lampyridae Rafinesque, 1815

Genus Petalacmis Olivier, 1908

Petalacmis wittmeri Reichardt, 1963: 108

Holotype: 1 đ. [on red] Holotipo; Ananindeua, Pará, Brasil, I.1963, Machado \& Pereira; Petalacmis wittmeri sp. N. Holótipo $\widehat{\partial}$, H. Reichardt det. 1963.

Conservation status: Good condition.

Lycidae Laporte, 1836

Genus Cartagonum Pic, 1922

Cartagonum mayense Nascimento \& Bocakova, 2010: 124

Paratype: 1 d. MEXICO: Chiapas, Palenque, 30.10.1978, E. Heiss; $\delta$; [on red] Cartagonum mayense n. sp., Nascimento \& Bocakova, 2009, PARATYPE.

Conservation status: Good condition; specimen glued on card by abdomen. Genitalia dissected into microvial with glycerin.

Cartagonum apiuba Nascimento \& Bocakova 2009: 125

Holotype: 1 ठ․ 01.XII.2007, Mata Sta. Tereza-EERP; Ribeirão Preto-SP-Brazil, EA Nascimento, CM Polegatto col.; ơ; [on red] Cartagonum apiuba n. sp., Nascimento \& Bocakova, 2009, HOLOTYPE. 
Conservation status: Good condition; specimen glued on card; last abdominal segments and left antenna glued on card under specimen; specimen glued on card by abdomen. Genitalia dissected into microvial with glycerin.

Paratypes: 3 o. 3.xi.2006, Mata Sta. Tereza-EERP; Ribeirão Preto-SP- Brazil, EA Nascimento, CM

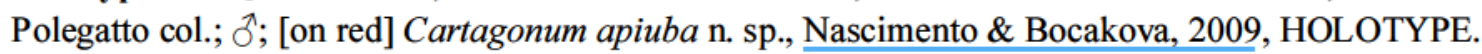

Conservation status: Good condition; specimens glued on cards; one with specimen right antenna missing; last abdominal segments and left antenna glued on card under specimen; Genitalia dissected into microvial with glycerin; one specimen with both terminal segments of antennae missing; ; last abdominal segments glued on card under specimen; Genitalia dissected into microvial with glycerin.; one specimen with terminal segments of one antenna glued on card under the specimen; genitalia Genitalia dissected into microvial with glycerin.

\section{Genus Lycomorphon Pic, 1922}

Lycomorphon amazonicum Nascimento \& Bocakova, 2009: 49

Holotype: 1 đ. Maturaca, AM, alto Rio Cauaburi, 28.XII.62-5.1.63, J. Bechyné col.; đ; [on red] Lycomorphon amazonicum n.sp., Nascimento \& Bocakova, 2009, HOLOTYPE.

Conservation status: Bad condition; specimen glued on card will legs and antennae missing; terminal segments of abdomen, part of one antenna and 3 legs glued on on card under the specimen; Genitalia dissected into microvial with glycerin.

Subgenus Spinolycus Nascimento \& Bocakova, 2009

Lycomorphon (Spinolycus) brasiliense Nascimento \& Bocakova, 2009: 50

Holotype: 1 ठ․ 01.XII.2006, Mata Sta. Tereza-ERRP, Ribeirão Preto-SP-Brazil, Nascimento EA, Polegatto CM col.; đ'; [on red] Lycomorphon (Spinolycus) brasiliense n.sp., Nascimento \& Bocakova, 2009, HOLOTYPE.

Conservation status: Bad condition; specimen glued on card with both antennae; terminal segments of one antenna glued on card under specimen; Genitalia dissected into microvial with glycerin.

\section{Phengodidae LeConte, 1861}

\section{Genus Zarhipis LeConte, 1881}

\section{Zarhipis tiemmani Linsdale, 1964: 253}

Paratype: $1 \delta$. [on red] Paratipo; 4 mi. east of Inyokern. Kern co. Calix, 3.v.48; D.L. Tiemman Collector; Paratype Zarhipis tiemanni Linsdale; Zarhipis tiemanni Linsdale; Unpublished mms. Dr. Donald D. Linsdale. Conservation status: Good condition.

Paratype: $1 \hat{\delta}$. [on red] Paratipo; 4 mi. N.E., Inyokern. Kern co. Calix, IV-20-1960; D.L. Tiemman Collector; Zarhipis tiemanni Linsdale.

Conservation status: Good condition.

\section{Genus Phrixothrix Olivier, 1909}

\section{Phrixothrix tiemanni Wittmer, 1970: 55}

Holotype: 1 ठ̊. São José dos Campos, SP-Brasil, 10.I.1970; [on red] Phrixotrix tiemanni Wittmer, det. W. Wittmer; Holotypus.

Conservation status: Good condition.

Paratypes : 2 đ̊. São José dos Campos, SP—Brasil, 10.I.1970; [on red] Phrixotrix tiemanni Wittmer, det. W. Wittmer; Paratypus. 
Conservation status: Good condition.

Paratype: $1 \widehat{\partial}$. São José dos Campos, São Paulo, Brasil, 17.I.63; [on red] Phrixotrix tiemanni Wittmer, det. W. Wittmer; Paratypus.

Conservation status: Idem.

Paratype: 1 $\delta$. Itu, São Paulo, Brazil, II.1.63; [on red] Phrixotrix tiemanni Wittmer, det. W. Wittmer; Paratypus.

Conservation status: Idem.

\section{Phrixothrix alboterminatus Wittmer 1963: 80}

Holotype: 1 ô. 1.1934 Goyaz, Campinas, Coll. H. Spitz; [on red] Phrixothrix alboterminatus Wittmer, det. W. Wittmer; Holotypus.

Conservation status: Good condition.

Phrixothrix vivianii Wittmer, 1992: 130

Holotype: $1 \hat{\delta}$. [on red] Holotypus; Faz. Sta. Cruz, MTS-Brazil, 20.12.1990, V. Viviani; Phrixothrix viviani Wittmer, det. W. Wittmer.

Conservation status: Good condition; with right antenna missing.

Paratype: $1 \delta$. [on red] Paratypus; Faz. Sta. Cruz, MTS-Brazil, 20.12.1990, V. Viviani; Phrixothrix viviani Wittmer, det. W. Wittmer.

Conservation status: Idem.

\section{Genus Mastinocerus Solier, 1849}

\section{Subgenus Mastinocerus Solier, 1849}

Mastinocerus (Mastinocerus) nigroapicalis travassosi Wittmer, 1963: 95

Holotype: 1 ô. [on red] Holotypus; II.29, São Paulo, Ypiranga, R. Spitz leg.; Mastinocerus nigroapicalis ssp travassosi Wittmer, det W. Wittmer.

Conservation status: Bad condition; left antenna missing.

Paratype: $1 \hat{\delta}$. [on red] Paratypus; Brooklin Paulista, São Paulo, SP—Brasil, 10.VII.961, L. Travassos Filho; $M$. nigroapicalis ssp travassosi Wittmer, det. W. Wittmer.

Conservation status: Bad condition, with both antennae missing the specimen. One antenna glued on a card under the specimen.

\section{Subgenus Paramastinocerus Wittmer, 1976}

Mastinocerus (Paramastinocerus) janeirensis Wittmer, 1976: 483

Holotype: $1 \hat{\delta}$.[on red] Holotypus; Rio de Janeiro-GB, (Floresta da Tijuca), IV.1966, M. Alvarenga col.; 0.80, 0.50; Mastinocerus (Paramastinocerus) janeirensis Wittmer, det. W. Wittmer.

Conservation status: Good condition; specimen glued on card by right elytra.

Paratype: $1 \hat{\delta}$. [on red] Paratypus; Corceu, 12.XI.58, Macaos; Mastinocerus (Paramastinocerus) janeirensis Wittmer, det. W. Wittmer; Guardado 2.

Conservation status: Good condition; specimen glued on triangle by meso- and metaventrite.

Mastinocerus (Paramastinocerus) pauloensis Wittmer, 1963: 94

Holotype: 1 $\delta^{\lambda}$. [on red] Holotype; 4.XI.1955, Barueri, São Paulo, 6820; K. Lenko—Leg.; M. pauloensis Wittmer, det. W. Wittmer.

Conservation status: Good condition. 
Ptorthodius lanei Wittmer, 1963: 415

Holotype: 1 đో. [on red] Holotypus; Coleção J. Lane; 8.VII.39; Ter. Amapá, Rio Amapari, J. Lane col.; Ptorthodius lanei Wittmer, det. W. Wittmer.

Conservation status: Bad condition; specimen glued on triangle by left elytra; right antenna missing.

Paratype: $1 \delta$. [on red] Allotypus; Coleção J. Lane; Ter. Amapá, Rio Amapari, J. Lane col.; 8.VII.39.

Conservation status: Idem.

Additional notes: Up to the present no winged female of Phengodidae has been found, so in this case, the specimen labeled as allotype must be considered a paratype of the same sex and not as a true allotype.

\section{Genus Brasilocerus Wittmer, 1963}

Brasilocerus impressicollis Wittmer, 1970: 56

Holotype: 1 đ. Estação Biológica de Boracéia, Mun. de Salesópolis, S.P., Brasil, 12.xi.69; X-Larva; Adult 18-IV-70; Brasilocerus impressicollis Wittmer, det. W. Wittmer; [on red] Holotypus.

Conservation status: Good condition.

Additional notes: Brasilocerus impressicollis is a junior synonym of Phrixothrix oberthuri Pic, 1955 that was transferred to Brasilocerus by Wittmer (1976:471). The Current name is Brasilocerus oberthuri (Pic, 1955).

Brasilocerus minasensis Wittmer, 1976: 472

Holotype: 1 ठ․ [on red] Holotype; Vila Monte Verde, Minas Gerais, 16.III.1966, J. Halik, 1706; Brasilocerus minasensis Wittmer, det. W. Wittmer.

Conservation status: Good condition; specimen glued on transparent triangle by abdominal segments.

Paratype: $1 \hat{\delta}$. [on red] Paratypus; Vila Monte Verde, Minas Gerais, 16.III.1966, J. Halik, 1706; Cantharidae; Phengodes sp.; Brasilocerus minasensis Wittmer, det. W. Wittmer; Guardado 1.

Conservation status: Idem.

\section{Genus Euryopa Gorham, 1881}

\section{Euryopa clarindae Wittmer 1996: 129}

Holotype: 1 đે. [on red] Holotypus; Cassilândia-MS, Faz. Olho d'Água, 02.XI.1986; E.J.H. Bechara, S. Ide; Coleta noturna, Luz vermelha em todo o corpo; Euryopa clarindae Wittmer, det. W. Wittmer; 809.

Conservation status: Bad condition; specimen glued on triangle; left antenna broken and right missing.

Additional notes: the third label of the specimen brings the following information (translated from Portuguese): night collect, red light around the body.

\section{Euryopa laurae Wittmer 1996: 129}

Holotype: 1 $\delta$. [on red] Holotypus; V. Viviani col., Faz. Sta. Cruz, Costa Rica. MS. Brazil; 14/12/1992 R2; Euryopa laurae Wittmer, det. W. Wittmer; 810.

Conservation status: Bad condition; specimen glued on card.

Rhinorhiphidae Lawrence, 1988

Genus Rhinorhipus Lawrence, 1988

Rhinorhipus tamborinensis Lawrence, 1988: 4 
Paratype: 1 đ. 27.56S 153 12E, Joalah N.P., Tamborine Mt. Q, 18-21. Oct. 1978, Lawrence \& Weir; On low vegetation.

Conservation status: Good condition.

\section{Acknowledgments}

I would like to thank Antonio Santos-Silva for his many valuable suggestions and corrections on the manuscript; Dr. Cleide Costa, Dr. Sonia Casari and Dr. Simone P. Rosa for providing literature for Elateridae beetles; Gabriel Biffi and Felipe F. Barbosa for providing literature, respectively, to Cantharidae and Eucnemidae; to Carlos Campaner and Ana Vasques for the support with Coleoptera Collection information; to Dione, Marta and Ricardo of the Library of MZSP by their helps with literature of other families; to Livia P. Prado for its suggestions on the style of the manuscript; to CAPES by the master's scholarship.

\section{References}

Aranda, S.G. (1996) Three new species and a redescription for the genus Aptopus Eschcholtz (Coleoptera, Elateridae) in Argentina. Acta Zoologica Lilloana, 42 (2), 433-445.

Barbosa, F.F., Quintino, H.Y. \& Ferreira, V.S. (2013) Checklist of the Immature Coleoptera Collection of the Museu de Zoologia da Universidade de São Paulo (CIC-MZSP) In: Fikáček, M., Skuhrovec, J. \& Šípek, P. (Eds.), Abstracts of the Immature Beetles Meeting 2013 October 3-4, Prague, Czech Republic. Acta Entomologica Musei Nationalis Pragae, 53 (2), 891-910.

Becker, E.C. (1956) Revision of the Nearctic Species of Agriotes (Coleoptera: Elateridae). Memoirs of the Entomological Society of Canada, 88 , Supplement 1, pp. 5-101.

Becker, E.C. (1971) Five new species of Megapenthes from the Southwestern United States, with a key to the Nearctic species (Coleoptera: Elateridae). The Canadian Entomologist, 103 (2), 145-167. http://dx.doi.org/10.4039/Ent103145-2

Becker, E.C. (1972) A new species of Anchastus from Arizona and Baja California (Coleoptera: Elateridae). The Coleopterists Bulletin, 26 (3), 121-123.

Becker, E.C. (1974) Revision of the Nearctic species of Athous (Coleoptera: Elateridae) East of the Rocky Mountains. The Canadian Entomologist, 106 (7), 711-758. http://dx.doi.org/10.4039/Ent106711-7

Bocakova, M., Bocak, L., Hunt, T., Teravainen, M. \& Vogler, A.P. (2007) Molecular phylogenetics of Elateriformia (Coleoptera), Evolution of bioluminescente and neoteny. Cladistics, 23, 477-496. http://dx.doi.org/10.1111/j.1096-0031.2007.00164.x

Bouchard, P., Bousquet, Y., Davies, A.E., Alonso-Zarazaga, M.A., Lawrence, J.F., Lyal, C.H.C., Newton, A.F., Reid, C.A.M., Schmitt, M., Slipinski, S.A., Smith, A.B.T. (2011) Family-group names in Coleoptera (Insecta). Zookeys, 88, 1-972. http://dx.doi.org/10.3897/zookeys.88.807

Brancucci, M. (1983) A new Paramonius from Brazil (Coleoptera: Cantharidae). The Coleopterists Bulletin, 37 (4), 362-364.

Branham, M.A. \& Wenzel, J.W. (2000) The evolution of Bioluminescence in Cantharoids (Coleoptera: Elateroidea). The Florida Entomologist, 84 (4), 565-586. http://dx.doi.org/10.2307/3496389

Brooks, A.R. (1960) Adul Elateridae of Southern Alberta, Saskatchewan and Manitoba (Coleoptera). The Canadian Entomologist, 92, supplement 20, pp. 5-63.

Brown, W.J. (1933) Studies in the Elateridae, I (Coleop.). The Canadian Entomologist, 65 (6), 133-141. http://dx.doi.org/10.4039/Ent65133-6

Brown, W.J. (1934a) The American Species of Dalopius Esch. (Elateridae, Coleop.). The Canadian Entomologist, 66 (2), $30-39$. http://dx.doi.org/10.4039/Ent6630-2

Brown, W.J. (1934b) The American Species of Dalopius Esch. (Elateridae, Coleop.). The Canadian Entomologist, 66 (3), $66-72$. http://dx.doi.org/10.4039/Ent6666-3

Brown, W.J. (1934c) The American Species of Dalopius Esch. (Elateridae, Coleop.). The Canadian Entomologist, 66 (4), $87-96$. http://dx.doi.org/10.4039/Ent6687b-4

Brown, W.J. (1934d) The American Species of Dalopius Esch. (Elateridae, Coleop.). The Canadian Entomologist, 66 (5), $102-110$. 
http://dx.doi.org/10.4039/Ent66102-5

Brown, W.J. (1935a) American species of Ludius; the aeripennis group. The Canadian Entomologist, 67 (6), $125-135$. http://dx.doi.org/10.4039/Ent67125-6

Brown, W.J. (1935b) American species of Ludius; the propola group. The Canadian Entomologist, 68 (8), $177-187$. http://dx.doi.org/10.4039/Ent68177-8

Brown, W.J. (1939) Some American species of Ludius (Coleoptera). The Canadian Entomologist, 71 (2), 4449. http://dx.doi.org/10.4039/Ent7144-2

Casari, S.A. (1996) Systematics and Phylogenetics analysis of Alaus Eschscholtz, 1829 (Coleoptera: Elateridae, Agrypninae, Hemirhipini). Revista Brasileira de Entomologia, 40(2), 249-298.

Casari, S.A. (1999) Three new species of Chacolepis Candezè, 1857 (Col. Elateridae: Agrypninae: Hemirhipini). Annales de la Société Entomologique de France, 35 (2), 203-215.

Casari, S.A. (2002) Review of the genus Chalcolepidius Eschscholtz, 1829 (Coleoptera, Elateridae, Agrypninae. Revista Brasileira de Entomologia, 46 (3), 263-428. http://dx.doi.org/10.1590/S0085-56262002000300007

Casari, S.A. (2003) New species of Alaus Eschscholtz, 1829 (Coleoptera: Elateridae, Agrypninae, Hemirhipini). Annales de la Société Entomologique de France, 39 (4), 315-333. http://dx.doi.org/10.1080/00379271.2003.10697390

Casari, S.A. (2009) A new species of Dicrepidius Eschscholtz from Brazil and new records for D. ramicornis (Palisot de Beuvois) (Coleoptera, Elateridae, Elaterinae). Revista Brasileira de Entomologia, 53 (1), 55-58. http://dx.doi.org/10.1590/S0085-56262009000100014

Casari, S.A. (2011) New species of Horistonotus Candèze from Brasil (Coleoptera, Elateridae, Cardiophorinae). Zootaxa, 3139, 1-27.

Casari, S.A. (2012) A new species and redescriptions of three species of Atractosomus Lacordaire from Brazil (Elateridae, Elaterinae). Revista Brasileira de Entomologia, 56 (4), 419-430. http://dx.doi.org/10.1590/S0085-56262012000400005

Casari, S.A. (2013) New genera of Dicrepidiina from the Neotropical Region (Coleoptera, Elateridae, Elaterinae, Ampedini). Zootaxa, 3721 (2), 143-156. http://dx.doi.org/10.11646/zootaxa.3721.2.2

Casari-Chen, S.A. (1986) Nova espécie do gênero Hapsodrilus Costa, 1975 (Hapsodrilini, Pyrophorinae, Elateridae). Revista Brasileira de Entomologia, 30 (2), 221-222.

Casari-Chen, S.A. \& Costa, C. (1992) A new species of Anoplischius Candèze, 1859, with Biological data (Coleoptera, Elateridae, Dicrepidiinae). Revista Brasileira de Entomologia, 36 (1), 47-54.

Chassain, J. (2005) Description de trois nouvelles espéces de Physodactylinae du Mato Grosso (Brésil) (Coleoptera, Elateridae). Boletín de la Sociedad Entomológica Aragonesa, 36, 65-71.

Chassain, J. (2009) Description d'une nouvelle espèce guyanaise et brésilienne d'élatéride du genre Coctilelater (Costa, 1975) (Coleoptera, Elateridae, Agrypninae, Pyrophorini). L'Entomologiste, 65 (1), 55-58.

Chassain, J. (2011) Description d'une espèce guyanaise nouvelle du genre Cardiorhinus (Coleoptera, Elateridae, Elaterinae, Agriotini), Le Coléoptériste, 14 (3), 207-210.

Cobos, A.S. (1955) Nueva aportación al conocimiento de los Eucnémidos (Insecta, Coleoptera) neotropicales: Colección R. Golbach. Bulletin de l'Institut Royal des Sciences Naturelles de Belgique, 31 (88), 1-12.

Cobos, A.S. (1956) Nuevos géneros y especies de Eucnemidae (Insecta, Coleoptera) de la fauna neotropical. Bulletin de l'Institut Royal des Sciences Naturelles de Belgique, 32 (41), 1-16.

Cobos, A.S. (1959) Coleópteros "Buprestidae" y "Elateridae" de la Isla Fernando de Noronha (Brasil). Revista Brasileira de Biologia, 19 (4), 436-470.

Constantin, R. (2010) A contribution to knowledge of the Cantharidae (Coleoptera, Elateroidea) in Ecuador and French Guiana. Entomologica Basiliensia et Collectionis Frey, 32, 7-29.

Costa, C. (1966) On a new genus of Hypolithinae (Col., Elateridae). Papéis Avulsos de Zoologia, 19 (23), $261-267$.

Costa, C. (1968) Descrição de uma espécie nova do gênero Anoplischius (Col., Elateridae). Papéis Avulsos de Zoologia, 21 (16), 161-164.

Costa, C. (1972) Gênero Pyrophorus. 6. Redescrição de P. tuberculifer Eschscholtz, P. phosporescens Castelnau e descrição de quatorze espécies novas (Col. Elateridae). Papéis Avulsos de Zoologia, 25 (21), 199-227.

Costa, C. (1973) Buckelater, gen. n. (Coleoptera, Elateridae, Cardiophorinae). Revista Brasileira de Entomologia, 17 (5), 33-36.

Costa, C. (1975a) Systematics and evolution of the tribes Pyrophorini and Heligmini with description of Campyxoleninae, new subfamily (Coleoptera, Elateridae). Arquivos de Zoologia, 26 (2), 49-191. http://dx.doi.org/10.11606/issn.2176-7793.v26i2p49-190

Costa, C. (1975b) Novas espécies do gênero Ptsimopsia Costa, 1975 (Coleoptera, Elateridae, Pyrophorinae). Papéis Avulsos de Zoologia, 29 (9), 55-64.

Costa, C. (1975c) Notas sobre o gênero Pyroptesis com descrição e dados de P. gilvus, sp. n. (Coleoptera, Elateridae, Pyrophorinae). Papéis Avulsos de Zoologia, 29 (1), 1-6.

Costa, C. (1978) Sistemática e Bionomia de Pyrearimus Costa, 1975 (Coleoptera, Elateridae). Arquivos de Zoologia, 29 (4), 
$185-236$.

http://dx.doi.org/10.11606/issn.2176-7793.v29i4p185-236

Costa, C. (1979) Novas espécies do gênero Hypsiophtalmus Latreille, 1834, e revalidação de Pyrearinus brevicollis (Eschscholtz, 1829), comb. n. (Coleoptera, Elateridae, Pyrophorinae). Papéis Avulsos de Zoologia, 32 (22), $261-276$.

Costa, C. (1980a) Novas espécies do gênero Lygelater Costa, 1975 (Coleoptera, Elateridae, Pyrophorinae). Papéis Avulsos de Zoologia, 33 (20), 299-307.

Costa, C. (1980b) Novas espécies dos gêneros Ignelater e Opselater (Coleoptera, Elateridae, Pyrophorinae). Papéis Avulsos de Zoologia, 33 (7), 157-176.

Costa, C. (1982) Pyrearinus termitilluminans, sp. n., with description of the immature stages (Coleoptera, Elateridae, Pyrophorini). Revista Brasileira de Zoologia, 1 (1), 23-30. http://dx.doi.org/10.1590/S0101-81751982000100003

Costa, C. (1991) New species of the genus Fulgeochlizus Costa, 1975 (Elateridae, Pyrophorinae). Revista Brasileira de Entomologia, 35 (3), 567-572.

Costa, C. (1999) Coleoptera. In: Brandão, C.R.F \& Cancello, E.M. (Eds.), Invertebrados Terrestres. V Biodiversidade do Estado de São Paulo. Síntese do Conhecimento ao inal do Século XX FAPESP, São Paulo, pp.113-122. [Joly, C.A. \& Bicudo, C.E.M. (Orgs.)]

Costa, C. (2010) The Immature Coleoptera Collection of The "Museu de Zoologia da Universidade de São Paulo" Brazil. In: Fikáček, M., Skuhrovec, J. \& Šípek, P. (Eds.), Abstracts of the Immature Beetles Meeting 2009, October 1-2, Prague, Czech Republic. Acta Entomologica Musei Nationalis Pragae, 50, pp. 324-325.

Costa, C., Ide, S., Rosado-Neto, G.H., Galileo, M.H.M., Vasconcelos da Fonseca, C.R., Valente, R.M., Monné, M.A. (2000) Diagnóstico del conocimiento de las Principales Colecciones Brasileñas de Coleoptera. In: Martín-Piera, F., Morrone, J.J. \& Melic, A. (Eds.), Hacia un Proyecto CYTED para el Inventario y Estimación de la Diversidad Entomológica en Iberoamérica, PrIBES-2000, 326 pp.

Costa, C., Vanin, S.A., Lawrence, J.F. \& Ide, S. (2003) Systematics and cladistic analysis of Cerophytidae (Elateroidea: Coleoptera). Systematic Entomology, 28, 372-407. http://dx.doi.org/10.1046/j.1365-3113.2003.00219.x

Germar, E.F. (1841) Beiträge zu einer Monographie der Gattung Pyrophorus. Zeitschrift für die Entomologie, 3, 1-76.

Golbach, R. (1983a) História del género Cardiorhinus Esch. 1829 (col. Elateridae). Acta Zoologica Lilloana, 37 (1), $35-54$.

Golbach, R. (1983b) Las especies de los grupos 3 y 4 del genero Cardiorhinus Eschscholtz. (Col. Elateridae). Acta Zoologica Lilloana, 37 (1), 153-177.

Golbach, R. (1983c) Primera cita de la familia Cerophytidae (Coleoptera) para Paraguay, Bolivia y la Argentina. Acta Zoologica Lilloana, 37, 131-139.

Golbach, R.A \& Aranda, S. (1991) Athous fueguensis n. sp., primer representante de la subfamilia Athouinae en la Argentina (Col. Elat.). Acta Zoologica Lilloana, 40 (1), 83-87.

Gurjeva, E.L. (1989) Fauna of USSR, Coleoptera, 12(3), Click-Beetles (Elateridae), Subfamily Athoinae, Tribe Ctenicerini. "Nauka" Publishing House, Leningrad, 293 pp.

Hunt, T., Bergsten, J., Levkanicova, Z., Papadopoulou, A., St. John, O., Wild, R., Hammond, P.M., Ahrens, D., Balke, M., Caterino, M.S., Gómez-Zurita, J., Ribera, I., Barraclough, T.G., Bocakova, M., Bocak, L. \& Vogler, A.P. (2007) A comprehensive Phylogeny of Beetles Reveals the Evolutionary Origins of a Superradiation. Science, 318, 1913-1916.

Johnson, P.J. (2002) Lectotype designations for Elateridae (Coleoptera) described by George C. Champion in the Biologia Centrali-Americana. Dugesiana, 9, 15-46.

Kundrata, R. \& Bocak, L. (2011) The phylogeny and limits of Elateridae (Insecta, Coleoptera): is there a commom tendency of click beetles to soft-bodiedness and neoteny? Zoologia Scripta, 40 (4), 364-378. http://dx.doi.org/10.1111/j.1463-6409.2011.00476.x

Kundrata, R., Bocakova, M. \& Bocak, L. (2014). The comprehensive phylogeny of the superfamily Elateroidea (Coleoptera: Elateriformia). Molecular Phylogenetics and Evolution, 76, 162-171. http://dx.doi.org/10.1016/j.ympev.2014.03.012

Lane, M.C. (1971) Family Elateridae [excl. Cardiophorinae], In: Hatch, M.H. (Ed.), Beetles of the Pacific Northwest. Vol. 5. University of Washington Press, Seattle, pp. 6-34.

Lawrence, J.F., Bocak, L., Bocakova, M., Beutel, R.G. \& Muona, J. (2010) 4. Elateroidea. In: Leschen, R.A.B., Beutel, R.G., Lawrence, J.F. (Eds.), Coleoptera, Beetles. Vol. 2. Morphology and Systematics (Elateroidea, Bostrichiformia, Cucujiformia partim). In: Kristensen, N.P., Beutel, R.G. (Eds.), Handbook of Zoology, Arthropoda: Insecta. Walter de Gruyter GmbH \& Co. KG, Berlin/New York, pp. 35-38.

Lawrence, J.F. (1988) Rhinorhipidae, a new beetle family from Australia, with comments on the phylogeny of the Elateriformia. Invertebrate Taxonomy, 2 (1987), 1-53.

Lawrence, J.F., Slipinski, A., Seago, A. E., Thayer, M. K., Newton, A.F. \& Marvaldi, A.E. (2011) Phylogeny of the Coleoptera Based on Morphological Characters of Adults and Larvae. Annales Zoologici, 61 (1), 1-217.

Linsdale, D.D. (1964) A revision of the genus Zarhipis LeConte (Coleoptera: Phengodidae). The Wasmann Journal of the Biology, 22 (2), 225-260.

Marinoni, R.C. \& Marinoni, L. (2012) Breve histórico da entomologia brasileira. In: Rafael, J.A., Melo, G.A.R., Carvalho, C.J.B., Casari, S.A., Constantino, R. (Org.), Insetos do Brasil. Editora Holos, Ribeirão Preto, pp. 2-20. 
Nascimento, E.A. \& Bocakova, M. (2009) A revision of the genus Lycomorphon (Coleoptera: Lycidae). Zootaxa, $2132,40-52$.

Nascimento, E.A. \& Bocakova, M. (2010) Review of the Neotropical genus Cartagonum (Coleoptera: Lycidae). The Canadian Entomologist, $142(2), 120-127$.

Navajas, E. (1944) Elateridae (Col.) de Monte Alegre com a descrição de uma espécie nova. Papéis Avulsos do Departamento de Zoologia, 6, 51-74.

Navajas, E. (1946) Sociedade Brasileira de Entomologia. In: Livro de homenagem a Romualdo Ferreira d'Almeida, editado para comemorar o $30^{\circ}$ aniversário de suas atividades Entomológicas. Sociedade Brasileira de Entomologia, São Paulo, pp. 349,19 plates.

Ohira, H. \& Becker, E.C. (1973) Elateridae (Coleoptera) from the Canadian Nepal expedition (1967). Descriptions of new species and records of the subfamilies Negastriinae and Hypnoidinae. Oriental Insects, 7 (1), 69-77.

Reichardt, H. (1963) Notas sobre el genero Petalacmis, con descripcíon de una especie nueva. Neotropica, 9, $107-110$.

Rosa, S.P. (2004) Revisão do gênero Opselater Costa (Coleoptera, Elateridae, Agrypninae). Revista Brasileira de Entomologia, $48(2), 203-219$.

Rosa, S.P. (2004) Nova espécie do gênero Ptsimopsia Costa (Coleoptera, Elateridae, Agrypninae). Revista Brasileira de Entomologia, $48(2), 227-228$.

Rosa, S.P. (2009) Description of Coctilelater minimus sp. nov. (Coleoptera, Elateridae, Agrypninae). Revista Brasileira de Entomologia, 53 (1), 36-37.

Rosa, S.P. (2010) New species of Ignelater Costa (Coleoptera, Elateridae, Pyrophorini). Papéis Avulsos de Zoologia, 50 (29), 445-449.

Rosa, S.P. (2011) New species of Triplonychus Candèze and Globothorax Fleutiaux from Brazil (Coleoptera, Elateridae, Cardiophorinae). Zootaxa, 2831, 1-22.

Rosa, S.P. \& Costa, C. (2009) Metapyrophorus pharolim a new genus and species of Pyrophorini (Coleoptera, Elateridae, Agrypninae). Revista Brasileira de Entomologia, 52 (1), 45-48.

Slipinski, S.A., Leschen, R.A.B. \& Lawrence, J.F. (2011) Order Coleoptera Linnaeus, 1758. In: Zhang, Z.Q. (Ed.), Animal biodiversity: An outline of higher-level classification and survey of taxonomic richness. Zootcxa, 3148, $203-208$.

Soares, B.A.M \& Peracchi, A.L. (1964) Sôbre a presença de cerofítidas no Brazil, com a descrição de duas novas espécies (Insecta: Coleoptera, Cerophytidae). Anais do Segundo Congresso Latino Americano de Zoologia, pp. $127-134$. [Departamento de Zoologia, Secretaria da Agricultura do Estado de São Paulo, São Paulo]

Stibick, J.N.L. (1968) A preliminary revision of the genus Tropihypnus (Coleoptera: Elateridae). Entomological News, 79, 168-187.

Stibick, J.N.L. (1980) A revision of the Hypnoidinae of the World (Col. Elateridae). Part 4. The Hypnoidinae of India. Section 12. Eos-Revista Espanola de Entomologia, 54, 247-273.

Taddei, V.A., Martins, U.R., Vivo, M., Percequillo, A.R. (1999) Museus e coleções zoológicas. In: Brito, M.C.W., Joly, C.A. (Org.), Biodiversidade do estado de São Paulo. 7 - Infra-estrutura para a conservação da Biodiversidade. Winner Graph, São Paulo, pp. 49-67.

Wittmer, W. (1945) Nuevos Cantharidae (Col.) (4 Contribucion al conocimiento de los Malacodermata neotropicais). Revista de la Sociedad Entomologica Argentina, 12, 313-326.

Wittmer, W. (1952) 13. Beitrag zur Kenntnis der Neotropischen Malacodermata (Coleoptera). Revista Chilena de Entomologia, 2, 197-205.

Wittmer, W. (1963) Zur Kenntnis der Phengodidae (Coleoptera) (25. Beitrag zuer Kenntnis der neotropischen Malacodermata). Mitteilungen der Schweizerischen entomologischen Gesellschaft, 36, 73-99.

Wittmer, W. (1966) 27. Beitrag zur Kenntnis der Neotropischen Malacodermata (Coleoptera). Studia Entomologica, 9, $411-416$.

Wittmer, W. (1967) 28. Beitrag zur Kenntnis der Neotropischen Malacodermata (Coleoptera). Studia Entomologica, 10, 419-432.

Wittmer, W. (1970) 30. Beitrag zur Kenntnis der neotropischen Malacodermata. Mitteilungen der Schweizerischen entomologischen Gesellschaft, 20, 55-59.

Wittmer, W. (1976) Arbeiten zu einer Revision der Familie Phengodidae (Coleoptera). Entomologische Arbeiten aus dem Museum G. Frey, 27, 415-524.

Wittmer, W. (1992) Zwei neue Phengodidae (Coleoptera) aus Brasilien. Mitteilungen der Schweizerischen entomologischen Gesellschaft, 42 (4), 130-132.

Wittmer, W. (1996) Ein weiterer Beitrag zur Kenntnis der Phengodidae (Coleoptera). Revista Brasileira de Entomologia, 40 (1), 125-129.

Woodruff, R.E. (2002) A new species of the beetle genus Brachypsectra from the Dominican Republic, with fossil conections (Coleoptera: Brachypsectridae). Insecta Mundi, 16 (4), 161-170. 